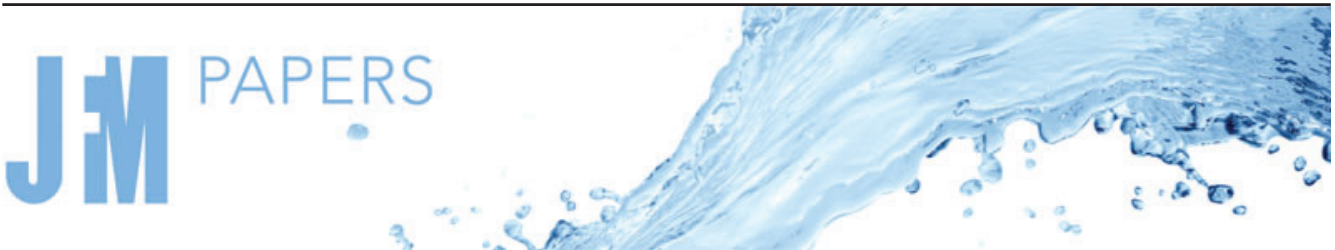

\section{Dynamics and length scales in vertical convection of liquid metals}

\author{
Lukas Zwirner ${ }^{1} \dagger$, Mohammad S. Emran ${ }^{1}$, Felix Schindler ${ }^{2}$, Sanjay Singh $^{2}$, \\ Sven Eckert ${ }^{2}$, Tobias Vogt $^{2}$ and Olga Shishkina ${ }^{1}$ \\ ${ }^{1}$ Max Planck Institute for Dynamics and Self-Organisation, Am Fassberg 17, 37077 Göttingen \\ ${ }^{2}$ Helmholtz-Zentrum Dresden-Rossendorf, Bautzner Landstr. 400, 01328 Dresden
}

(Received 16 April 2021; revised 7 October 2021; accepted 1 November 2021)

Using complementary experiments and direct numerical simulations, we study turbulent thermal convection of a liquid metal (Prandtl number $\operatorname{Pr} \approx 0.03$ ) in a box-shaped container, where two opposite square sidewalls are heated/cooled. The global response characteristics like the Nusselt number $N u$ and the Reynolds number $R e$ collapse if the side height $L$ is used as the length scale rather than the distance $H$ between heated and cooled vertical plates. These results are obtained for various Rayleigh numbers $5 \times 10^{3} \leq R a_{H} \leq$ $10^{8}$ (based on $H$ ) and the aspect ratios $L / H=1,2,3$ and 5. Furthermore, we present a novel method to extract the wind-based Reynolds number, which works particularly well with the experimental Doppler-velocimetry measurements along vertical lines, regardless of their horizontal positions. The extraction method is based on the two-dimensional autocorrelation of the time-space data of the vertical velocity.

Key words: convection in cavities

\section{Introduction}

The understanding of turbulent thermal convection is of great importance for astrophysics, geophysics, climate research and engineering purposes alike. Although convection has been under investigation for centuries, even today researchers struggle to unveil its complex nature. Usually, to study turbulent thermal convection, a model system is considered, where the fluid is confined between horizontal plates heated from below and cooled from above, commonly known as Rayleigh-Bénard convection (RBC) (Bodenschatz, Pesch \& Ahlers 2000; Ahlers, Grossmann \& Lohse 2009; Chillà \& Schumacher 2012). In this work we study a different model system, where the fluid

$\dagger$ Email address for correspondence: lukas.zwirner@ds.mpg.de 


\section{Zwirner and others}

is confined between vertical heated/cooled plates, also known as convection inside a differentially heated enclosure, side-heated convection or vertical convection. Throughout this work, we will refer to this system as vertical convection (VC).

$\mathrm{RBC}$ and $\mathrm{VC}$ are two limiting cases of the more general type of inclined convection (Daniels, Wiener \& Bodenschatz 2003; Chillà et al. 2004; Sun, Xi \& Xia 2005; Ahlers, Brown \& Nikolaenko 2006; Weiss \& Ahlers 2013; Shishkina \& Horn 2016; Zwirner \& Shishkina 2018; Zwirner et al. 2020a) and therefore are of particular importance. Note, that $\mathrm{VC}$, unlike $\mathrm{RBC}$, is inherently unstable even for the smallest temperature difference between the plates.

Batchelor (1954) was one of the first to investigate VC for very small temperature differences, and, in the beginning, the main interest was in studying the heat transport through double layer windows. Therefore, the studied geometry was of very large aspect ratio $L_{z} / H$, where $L_{z}$ is the height of the convection cell and $H$ is the distance between the heated and cooled vertical plates. Fujii et al. (1970) performed a detailed experimental study on the evolution of boundary layers (BLs) and local heat transport in VC, using two concentric cylinders, where the inner one was heated and the outer one cooled, and using oil and water as working fluids. Belmonte, Tilgner \& Libchaber (1995) used a cubic cell and different gases of Prandtl number $P r \approx 0.7$ for Rayleigh numbers $R a_{H} \leq 10^{11}$ and found by shadowgraph visualization a stably stratified bulk, while the temperature fluctuations were mainly observed close to the boundaries. Additionally, they measured a scaling of the thermal BL thickness $\sim R a^{-0.29}$. Koster, Seidel \& Derebail (1997) used $\mathrm{X}$-ray radiography to measure and visualize the density distribution inside a narrow VC cell filled with liquid gallium. This was a milestone in the visualization of liquid metal flows, however, it is only applicable to narrow cavities and also no conclusions about turbulent convection could be drawn. Braunsfurth et al. (1997) conducted experiments with liquid gallium inside a long VC cells of aspect ratios $L / H=1 / 3$ and $1 / 4$, for small Grashof numbers $\left(\operatorname{Ra} / \mathrm{Pr}<5 \times 10^{4}\right)$ and compared the results with two-dimensional numerical simulations. Here, we investigate flow at much larger $R a$ and large aspect ratios.

As in RBC (Shishkina 2021), in VC, the aspect ratio of the container influences the flow in a finite fluid layer confined between two differently heated plates (Batchelor 1954; Bejan 1980, 1985, 2013; Paolucci 1994). However, the effects of the container's size and the distance between its walls have not been investigated separately. Understanding this phenomenon is of special significance in convection, especially in a small- $\operatorname{Pr}$ fluid, as both these length scales influence the flow field. In particular, this sort of flow configuration is typically encountered in liquid metal batteries (Kelley \& Weier 2018) - a technology with potential in grid-scale storage. In this paper, we attempt to disentangle the aspect ratio dependency, and shift the focus onto the two predominant length scales using numerical and experimental data across several aspect ratios. Additionally, we also present a robust new technique which is capable of computing the Reynolds number based on the wind velocity.

There are only a few examples of ongoing investigations of $\mathrm{VC}$ and in addition to Churchill \& Chu (1975); Graebel (1981); Tsuji \& Nagano (1988); Chen \& Pearlstein (1989); Paolucci (1990); Versteegh \& Nieuwstadt (1999); Pallares et al. (2010); Kis \& Herwig (2012) and Wang et al. (2021); there are even more studies, but most of them use air, oil or water with relatively large Prandtl numbers, $\operatorname{Pr} \gtrsim 0.7$. However, recently, liquid metals with very small Prandtl numbers, $\operatorname{Pr} \ll 1$, have become a particular focus of investigations in the thermal convection community (King \& Aurnou 2013; Scheel

\& Schumacher 2016; Schumacher et al. 2016; Aurnou et al. 2018; Vogt et al. 2018a; Zwirner \& Shishkina 2018; Zürner et al. 2019; Zwirner et al. 2020a; Zwirner, Tilgner \& 


\section{Vertical convection of liquid metals}

Shishkina 2020b). The aim of the present work is to shed more light on VC of low-Prandtl-number fluids.

One important step that led to a better understanding of RBC was the development of the scaling theory by Grossmann \& Lohse $(2000,2002)$ (GLT). This theory is based on the exact relationships between the heat transport, represented by the dimensionless Nusselt number $N u$, and the kinetic and thermal dissipation rates. The GLT assumes various scaling regimes $\left(N u \sim R a^{\gamma}\right)$ depending on which part of the flow determines the scalings of the dissipation rates: the BLs or the bulk. This theory also concludes that there is no simple scaling law applicable for the entire range of Rayleigh numbers, but that the exponent $\gamma$ changes smoothly within these regimes. A similar approach has been applied by $\mathrm{Ng}$ et al. (2015) to $\mathrm{VC}$, however, the difficulty in this case is a non-closed term in the relationship between $\mathrm{Nu}$ and the kinetic energy dissipation rate. Nevertheless, $\mathrm{Ng}$ et al. (2015) concluded that a similar approach to the GLT is applicable to VC, when a suitable closure model for that specific term is found.

Although one might deem the question about which scales determine the flow settled, there is still active research on the most suitable scales. For example, Wei (2020) found the proper scale for the Reynolds shear stress in a differentially heated vertical channel is a mixed scale of the friction and the maximum mean velocity.

Scaling relations for heat transport and BL thicknesses in vertical convection have been investigated in the past (Batchelor 1954; Gill 1966; Saville \& Churchill 1969; Ostrach 1972). A recent approach agrees upon the same exponents for vertical convection in the laminar regime, there the exponents were derived from BL theory and confirmed by direct numerical simulations (DNS) in a theoretical work (Shishkina 2016).

It was found that for $\mathrm{Pr} \ll 1$ the Nusselt number and the wind-based Reynolds number scale with respect to the Rayleigh and Prandtl numbers as

$$
N u \sim \operatorname{Pr}^{1 / 4} \operatorname{Ra}^{1 / 4} \text { and } \operatorname{Re}_{\text {wind }} \sim \operatorname{Pr}^{-1 / 2} \operatorname{Ra}^{1 / 2},
$$

respectively. Note that the scaling relations are valid regardless of the length scale chosen and therefore the indices $H$ and $L$ are omitted. However, the pre-factor depends on this choice and in the following we show that the data collapse for different aspect ratios when the scale is chosen appropriately.

The remaining article is structured as follows: in $\S 2$ we introduce the experimental and numerical set-up, in $\S 3$ we present a detailed comparison of the experimental and numerical results and discuss the relevant length scale, heat and momentum transport and finally we conclude with $\S 4$.

\section{Experimental and numerical methods}

Here, we present our numerical and experimental methods. A sketch of the basic set-up used in the experiments and the three-dimensional DNS is shown in figure 1. We use a rectangular cell with variable aspect ratio, $L / H$, where the heated and cooled plates are squares of length $L=L_{y}=L_{z}$. The distance between the heated and cooled plates is $H$.

\subsection{Numerical set-up}

The incompressible Navier-Stokes equations in Oberbeck-Boussinesq approximation

$$
D_{t} \boldsymbol{u}=v \nabla^{2} \boldsymbol{u}-\nabla p+\alpha g\left(T-T_{0}\right) \hat{z}
$$




\section{Zwirner and others}

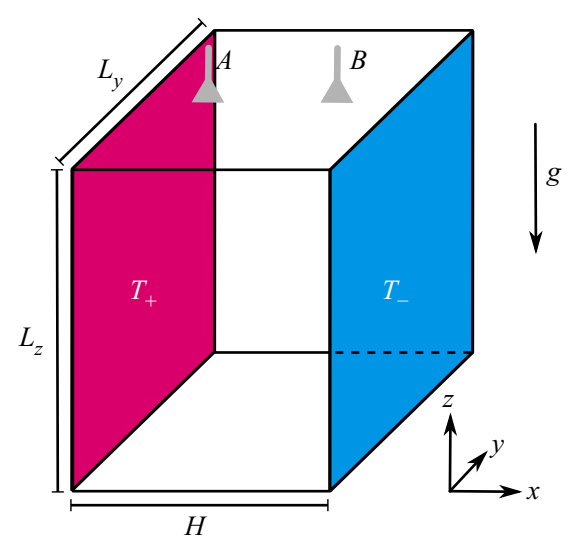

Figure 1. Sketch of the VC cell with a heated wall at the left side (temperature $T_{+}$) and cooled wall at the right side (temperature $T_{-}$). The velocity is measured along two lines parallel to the $z$-direction, using the Doppler probes $A$ and $B$. The direction of gravity is indicated by $g$. This sketch represents the set-up for the DNS as well as the experiments.

$$
\begin{gathered}
D_{t} T=\kappa \nabla^{2} T, \\
\nabla \cdot \boldsymbol{u}=0
\end{gathered}
$$

are solved using the high-order finite volume code GoLDFISH (Kooij et al. 2018) in Cartesian coordinates. Here, $D_{t}$ denotes the substantial derivative, $\boldsymbol{u}=\left(u_{x}, u_{y}, u_{z}\right)$ the velocity vector field, $p$ is the reduced kinetic pressure, $T$ the temperature, $T_{+}$the temperature of the hot vertical plate, $T_{-}$the temperature of the cold vertical plate, $T_{0}$ is the mean temperature $\left(T_{+}+T_{-}\right) / 2, v$ is the kinematic viscosity and $\kappa$ the thermal diffusivity. The equations are transformed into their non-dimensional representation using the distance $H$ between the hot and cold plates, the time $t_{f} \equiv H(\alpha g H \Delta)^{-1 / 2}$ and the temperature difference $\Delta \equiv T_{+}-T_{-}, g$ is the gravitational acceleration. Thus, our VC system depends on the following three non-dimensional input parameters:

$$
R a_{H} \equiv \alpha g \Delta H^{3} /(\kappa v), \quad \operatorname{Pr} \equiv \nu / \kappa, \quad \Gamma=L / H,
$$

which are the Rayleigh number, Prandtl number and aspect ratio, respectively. All DNS are conducted at $\operatorname{Pr}=0.03$.

The computational mesh, used in the simulations, is clustered near the boundaries and the largest simulation $\left(R a_{H}=10^{8}\right.$ and $\left.\Gamma=1\right)$ has a resolution of $674^{3}$ points, while the averaging time is usually $t_{\text {avg }} \geqslant 150 t_{f}$. We start to collect the statistics after the flow reaches a stationary state as indicated by the fluctuations of $N u(t)$ around a constant long-time average. The number of points within the viscous BL is always $N_{\delta_{u}} \geqslant 4$ and due to the small Prandtl number, the thermal BL is much thicker than the viscous one and therefore both BLs are well resolved. Additionally, we conducted a mesh convergence study and also ensure that our typical mesh distance $h$ resolves the Kolmogorov scale $\eta=$ $\left(v^{3} / \varepsilon_{u}\right)^{1 / 4}$ (where $\varepsilon_{u}$ is the kinetic energy dissipation rate), i.e. $h \lesssim \eta$, which represents the smallest scales of the flow. A detailed list of the DNS resolution can be found in the Appendix, table 1 .

Important characteristic output quantities in VC are the Nusselt number, the Reynolds number and the thermal/viscous BL thicknesses. The Nusselt number represents the heat transport through the system and is defined as

$$
N u_{H} \equiv \frac{\left\langle\overline{u_{x} T}-\kappa \partial_{x} \bar{T}\right\rangle_{A_{y z}}}{\kappa \Delta / H},
$$


where $\langle\cdot\rangle_{A_{y z}}$ means averaging over a slice parallel to the hot plate and ${ }^{-}$averaging over time. The thickness of the thermal BL is defined as usual: $\delta_{\theta} \equiv H /(2 N u)$. For the definition of the Reynolds number, we follow Shishkina (2016) and introduce the wind velocity in a similar manner as

$$
U_{\text {wind }} \equiv \max _{x}\left\{\left\langle\overline{u_{z}}\right\rangle_{A_{y z}}\right\}
$$

and with this the wind-based Reynolds number

$$
R e_{\text {wind }}=\frac{L U_{\text {wind }}}{v} .
$$

Furthermore, we define the thickness of the viscous BL using the slope method (Zhou \& Xia 2010)

$$
\delta_{u} \equiv \frac{U_{\text {wind }}}{\left.\partial_{x}\left\langle\overline{u_{z}}\right\rangle_{A_{y z}}\right|_{x=0}} .
$$

Note that, for laminar flow, $\delta_{u} \sim R e^{-1 / 2}$, and using (1.1) we conclude that $\delta_{u} \sim R a^{-1 / 4}$. For an example of the horizontal profile $\left\langle\overline{u_{z}}\right\rangle$ we refer the reader to figure $8(c, d)$.

\subsection{Laboratory set-up}

Figure 1 shows a schematic drawing of the experimental set-up. The experiments were performed in two rectangular vessels with a square vertical cross-section of $L^{2}=$ $200 \mathrm{~mm} \times 200 \mathrm{~mm}$ and a distance between the vertical heated/cooled boundaries of $H=$ 40 and $H=66 \mathrm{~mm}$, which gives aspect ratios of $\Gamma=L / H=5$ and $\Gamma \approx 3$, respectively. On the two square vertical surfaces of the vessel, the heat is introduced and removed via two copper plates, which are tempered by a circulating water bath and the temperature is controlled via thermocouples inside the copper plates.

The maximal thermal power input is $P=1500 \mathrm{~W}$ in this study. The other sidewalls are made of $30 \mathrm{~mm}$ thick Polyvinylchloride. To minimize heat losses, the whole convection cell is wrapped in a $30 \mathrm{~mm}$ thick closed-cell foam, which has a thermal conductivity of approximately $0.036 \mathrm{~W} \mathrm{mK} \mathrm{m}^{-1}$ and is $\sim 660$ times lower that of the liquid metal $\left(24 \mathrm{~W} \mathrm{mK}^{-1}\right)$.

The vessel is filled with the eutectic alloy GaInSn that has a melting temperature of $T_{S}=$ $10.5^{\circ} \mathrm{C}$. At room temperature, it has the density $\rho=6350 \mathrm{~kg} \mathrm{~m}^{-3}$, the thermal expansion coefficient $\alpha=1.24 \times 10^{-4} 1 \mathrm{~K}^{-1}$, the thermal conductivity $\lambda=24.05 \mathrm{~W} \mathrm{mK}^{-1}$, the kinematic viscosity $v=3.38 \times 10^{-7} \mathrm{~m}^{2} \mathrm{~s}^{-1}$ and the thermal diffusivity $\kappa=$ $1.05 \times 10^{-5} \mathrm{~m}^{2} \mathrm{~s}^{-1}$ (Plevachuk et al. 2014). The corresponding Prandtl number is $\operatorname{Pr} \approx 0.03$.

For the majority of the measurements, we keep the mean fluid temperature constant at approximately $21^{\circ} \mathrm{C}$. Only for the highest $R a$ do we have to increase the mean fluid temperature up to $35^{\circ} \mathrm{C}$. However, the temperature dependence of the material parameters is comparatively low, thus the Prandtl number changes only by approximately $10 \%$ $\left(2.97 \leq \operatorname{Pr} / 10^{-2} \leq 3.25\right)$. Therefore, we consider possible non-Oberbeck-Boussinesq effects to have a rather weak influence on the flow.

\subsection{Measuring technique}

In this study, the flow velocities are measured using ultrasonic Doppler velocimetry (UDV) which is an established measurement technique for opaque fluids like liquid metals (Aubert et al. 2001; Brito et al. 2001; Eckert \& Gerbeth 2002; Gillet et al. 2007; Nataf et al. 2008; 


\section{Zwirner and others}

Vogt, Räbiger \& Eckert 2014; Vogt et al. 2018a,b; Zürner et al. 2019, 2020; Yang, Vogt \& Eckert 2021; Vogt, Horn \& Aurnou 2021a; Vogt et al. 2021b). The used UDV system is a DOP3010 (from Signal Processing SA, Lausanne) equipped with $8 \mathrm{MHz}$ transducers. The UDV transducers send a pulsed ultrasonic signal into the liquid metal which is reflected by microscopic particles such as oxides. The position and velocity of the particles can be determined from the transit time of the ultrasound and the phase shift of the echo from subsequent echo pulses. This allows us to determine the beam-parallel velocity distribution along the ultrasonic beam (Takeda 2012). The two ultrasonic sensors used in this study measure velocities along the vertical direction (cf. figure 1). The distances from the sensors to the heated plate are $x_{p} / H=0.25$ and 0.75 for $\Gamma=5$, and $x_{p} / H=0.15$ and 0.85 for the $\Gamma=3$. The measuring system records velocity profiles with a time resolution of approximately $0.3 \mathrm{~s}$. The spatial resolution is approximately $1 \mathrm{~mm}$ in the beam direction and $5 \mathrm{~mm}$ in the lateral direction due to the diameter of the ultrasound emitting piezoelectric transducer.

The experiment is equipped with 22 thermocouples with nine being embedded in each copper plate to measure the temperature drop across the fluid layer. The remaining four thermocouples are attached to the water channels that temperate the copper plates, in order to determine the temperature change of the circulating water $\left(T_{\text {in }}-T_{\text {out }}\right)$. Together with the measurements of the water flow rate $\dot{V}$, this enables the calculation of the convective heat transport, which is expressed non-dimensionally by the Nusselt number

$$
N u=\frac{\dot{Q}_{\text {tot }}}{\dot{Q}_{\text {cond }}}=\frac{\rho c_{p} \dot{V}\left(T_{\text {in }}-T_{\text {out }}\right)}{\lambda L^{2} \Delta / H},
$$

where $c_{p}$ is the isobaric heat capacity of water. There are small differences between the measurements at the hot and cold plates due to thermal losses. The Nusselt numbers presented here are the averages of the hot and cold measurement $(\Gamma=5)$, or shown separately $(\Gamma=3)$.

\subsection{Extraction of the Reynolds number}

One objective of this work is to compare the simulations with the experiments, and therefore we need to define a Reynolds number based on a certain velocity that is easily measurable in both simulations and experiments. In the experiments, only limited velocity data are available from the UDV probes, while the simulations provide access to the complete velocity fields. Therefore, we can directly compare the two-dimensional velocity fields $u_{z}(t, z)$ only at the Doppler probe positions $\left(x_{p} / H=1 / 4\right.$ and $\left.3 / 4\right)$. Examples of these data are visualized in figure $2(a, c, e)$. There are multiple possibilities to define a characteristic velocity based on these data. Here, we investigate two candidates in detail, a straightforward one (the maximal velocity) and a more sophisticated one (based on the autocorrelation) which we define in the following.

The maximal velocity is defined as

$$
U_{\max } \equiv \max _{z}\left\{\overline{u_{z}(t, z)}\right\}
$$

and the corresponding Reynolds number

$$
R e_{L}^{\max } \equiv \frac{L U_{\max }}{v}
$$


Vertical convection of liquid metals

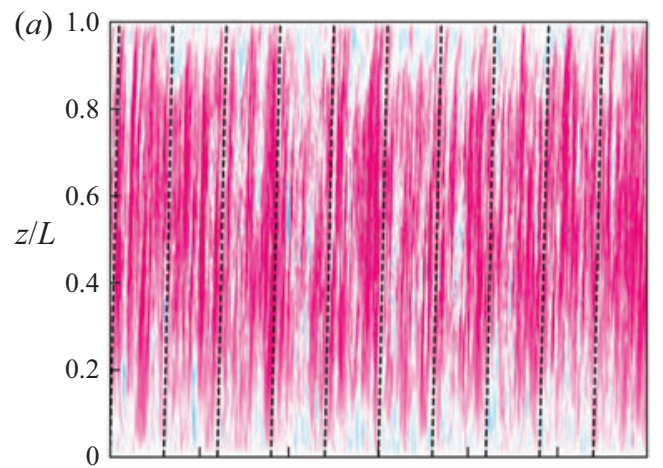

(b)

(a)
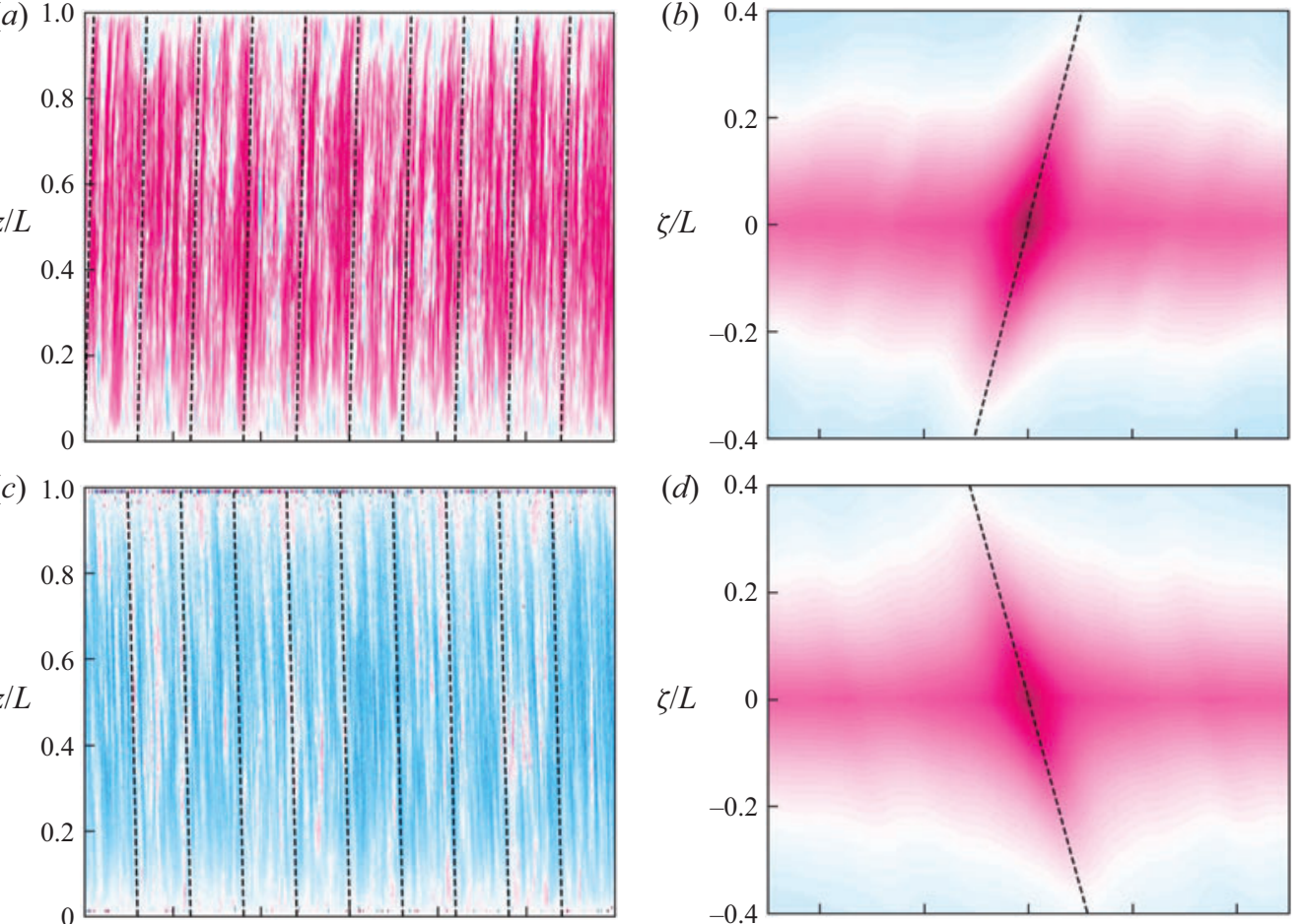

(d)
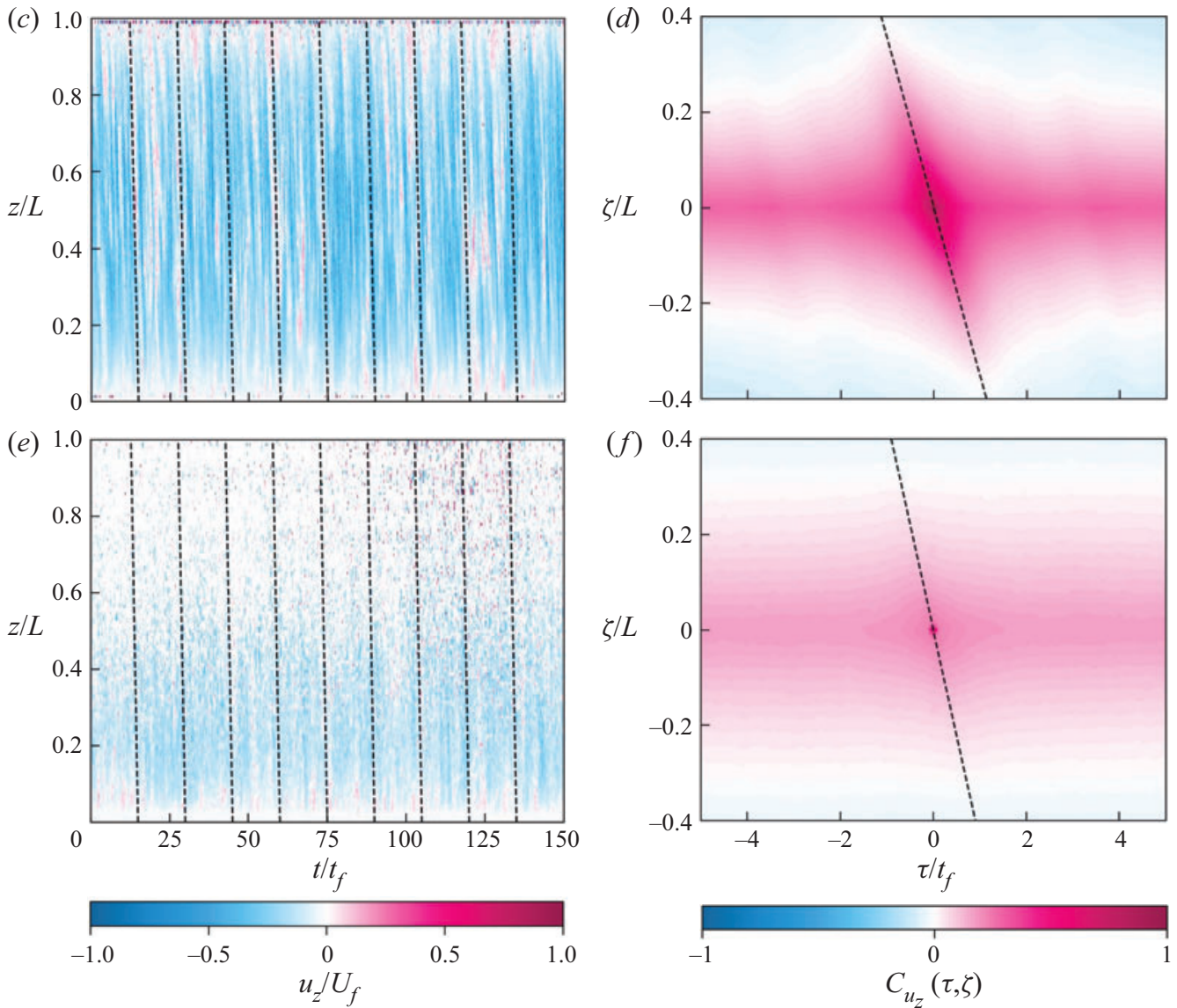

Figure 2. Examples of time-space plots of the vertical velocity $u_{z}(t, z)$ for: $(a)$ DNS data at probe $A, \Gamma=5$, $R a_{L}=1.25 \times 10^{7},(c)$ experimental data at probe $B, R a_{L}=1.05 \times 10^{7}$ and (e) noisy experimental data at probe $B, R a_{L}=6.94 \times 10^{6}$. (b,d,e) The autocorrelation function $\mathcal{C}_{u_{2}}(\tau, \zeta)$ for $(a, c, e)$ respectively, cf. (2.12). The dashed lines indicate the characteristic velocity $U^{\star}$ obtained from the autocorrelation as described in $\S 2.4$.

A more sophisticated approach is based on the two-dimensional autocorrelation of $u_{z}(t, z)$, which we calculate in a discrete manner as

$$
\mathcal{C}_{u_{z}}(\tau, \zeta)=\frac{1}{N M \sigma^{2}} \sum_{i, j}^{N, M} u_{z}^{\prime}\left(t_{i}, z_{j}\right) \cdot u_{z}^{\prime}\left(t_{i}-\tau, z_{j}-\zeta\right),
$$




\section{Zwirner and others}

where $u_{z}^{\prime}(t, z) \equiv u_{z}(t, z)-\left\langle\overline{u_{z}}\right\rangle_{z}$ and $\sigma$ is the standard deviation of $u_{z}$. Note that, in (2.12), the fraction in front of the sum normalizes $\mathcal{C}_{u_{z}}$ to values between -1 and 1 . Examples of $\mathcal{C}_{u_{z}}(\tau, \zeta)$ are shown in figure $2(b, d, f)$. The next step is to fit a straight line,

$$
\zeta(\tau)=U^{\star} \tau
$$

through the points

$$
\left\{\left(\tau_{i}, \zeta_{i}\right): \mathcal{C}_{u_{z}}\left(\tau_{i}, \zeta_{i}\right)=\max _{\tau} \mathcal{C}_{u_{z}}\left(\tau, \zeta_{i}\right)\right\}
$$

The slope of this line gives the characteristic velocity $U^{\star}$. Additionally, we restrict the domain of the fit to the relevant points around the origin. How this extraction algorithm works with experimental and numerical data is shown by several examples in figure 2 . At this point, we can define the Reynolds number based on $U^{\star}$ as

$$
R e_{L}^{\star}=\frac{L U^{\star}}{v}
$$

The advantage of evaluating the velocity in this way is that this method provides very robust results even in the case of low-quality signals. Figure 2(e) shows an example of a measurement with a very noisy signal. This is due to weak echo amplitudes caused by an imperfect acoustic contact between the transducer and the liquid metal or an insufficient number of reflective tracers in the measurement volume. In this case, the local velocity values are subject to a high measurement uncertainty, in particular with increasing distance from the sensor. However, the method proposed here uses almost all the available information from the measurements, and by taking into account the spatio-temporal nature of the velocity field, the deficit of poor signal quality can be essentially compensated.

\section{Results and discussion}

In the following, we will present the results and discuss the evidence, that the relevant length scale in VC is based on the plate size rather than on the distance between the hot and cold plates. Furthermore, we are going to explore the flow structures and BLs, and finally we discuss the new method based on the autocorrelation to determine Reynolds numbers from UDV data. A summary of the measured quantities can be found in the Appendix, tables 2 and 3, however, this is only for reference, as all the quantities are presented in various figures.

\subsection{Global flow organization}

To provide a deeper insight into the general flow organization, we show in figure 3 instantaneous flow fields at different $R a_{L}$ and aspect ratios $\Gamma$. We calculate the full heat transport vector

$$
\boldsymbol{\Omega} \equiv(\boldsymbol{u} T-\kappa \nabla T) /(\kappa \Delta / H),
$$

and then find regions where its magnitude is greater or equal to the Nusselt number $(|\boldsymbol{\Omega}| \geqslant N u)$. This prominently shows the large-scale circulation and captures the major regions, where heat is transported. Here, we choose the magnitude of the full heat flux vector as the basis and not just the $x$ component. In this way we can capture the regions of vertical heat transport close to the tempered walls and also the heat transport between them. Additionally, this surface is coloured by temperature, to distinguish the hot and cold streams of the flow. This way of visualization helps to overcome two difficulties: on the one 
(a)

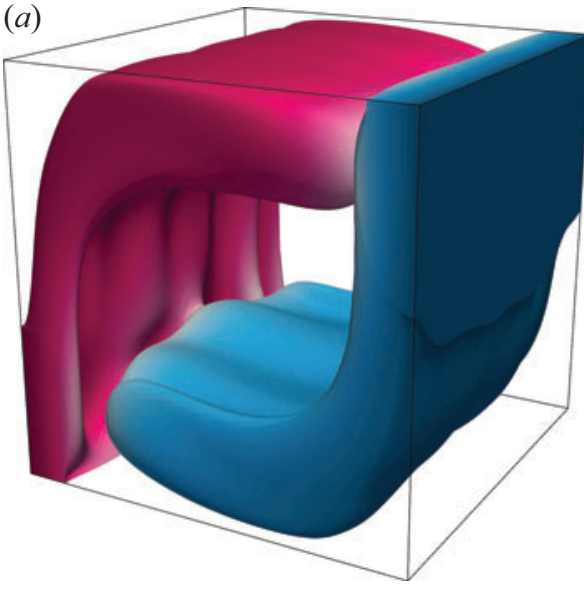

(d)

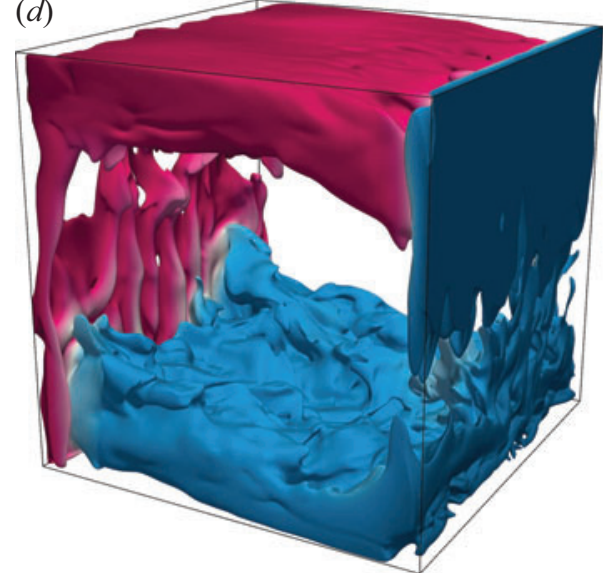

(b)

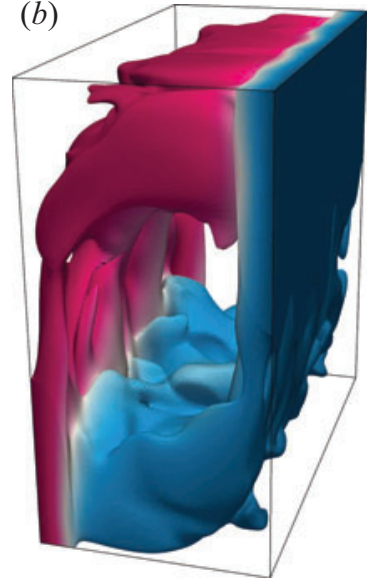

(e)

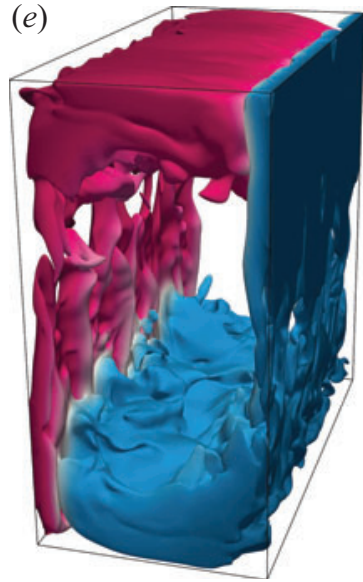

(c)

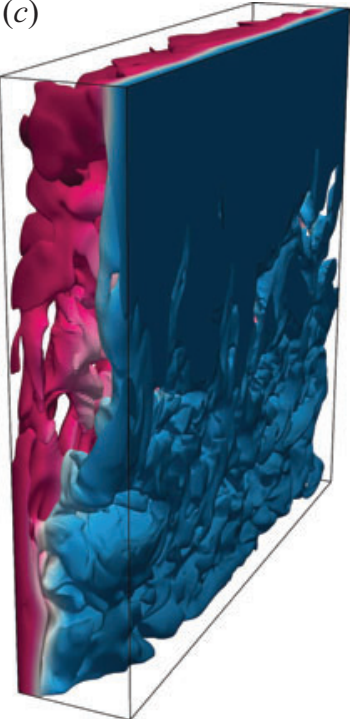

$T_{-}$

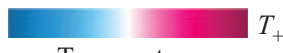

Figure 3. Instantaneous heat transport and temperature. The shown superstructures enclose regions, where the instantaneous heat transport $|\boldsymbol{\Omega}(t)| \geqslant N u$, and they are coloured with the local temperature. Panels $(a-c)$ are at $R a_{H}=5 \times 10^{4}$ and different aspect ratios $\Gamma=1,2,5$, respectively. Panels $(d, e, c)$ are at similar $R a_{L}=$ $5 \times 10^{6}, 4 \times 10^{6}, 6.25 \times 10^{6}$, respectively.

hand, vector fields are difficult to render in three dimensions and even streamlines appear rather cluttered, and on the other hand, temperature isosurfaces appear rather smooth due to the low Prandtl number and therefore hide the vigorous nature of turbulent convection in liquid metals.

\subsection{The characteristic length scale}

There are two prominent length scales in a rectangular box with square hot/cold plates: the distance $H$ between the plates and the height of the side hot/cold square plates $L$. One of the questions we are going to answer in this section is: On which length scale $(H$ or $L)$ should the scaling dimensionless quantities like $R a$ and $R e$ be based?

We denote quantities that are based on $L$ or $H$ with the subscript $L$ or $H$, respectively, and they convert as

$$
R a_{L}=R a_{H} \Gamma^{3}, \quad N u_{L}=N u_{H} \Gamma \quad \text { and } \quad R e_{L}=\operatorname{Re}_{H} \Gamma .
$$




\section{Zwirner and others}

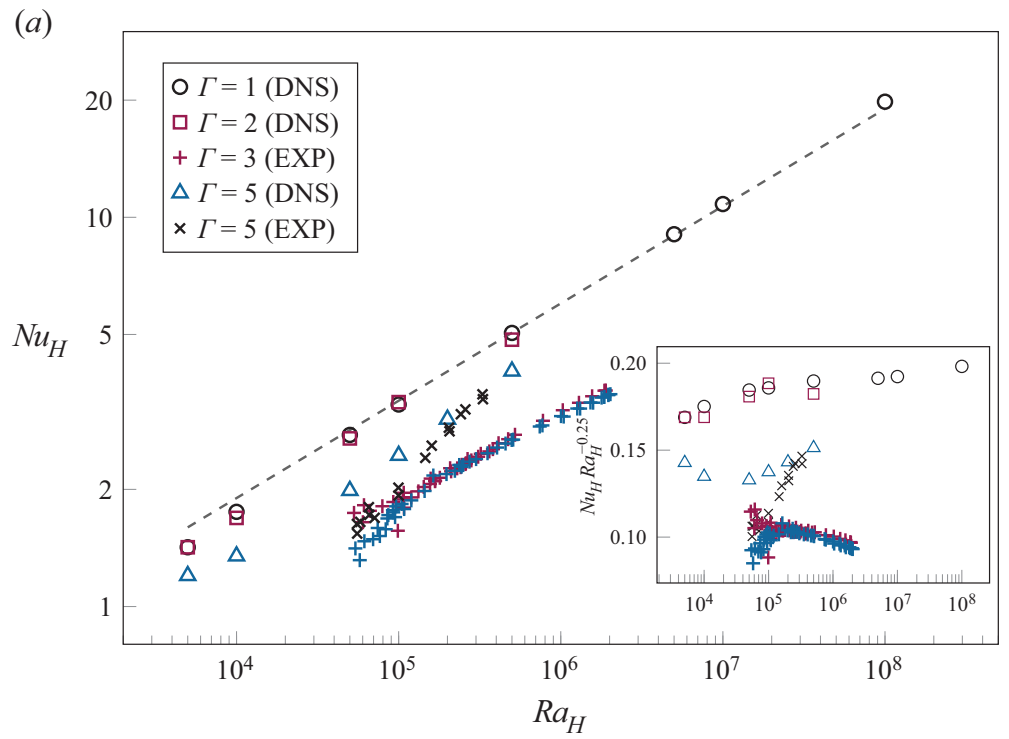

(b)

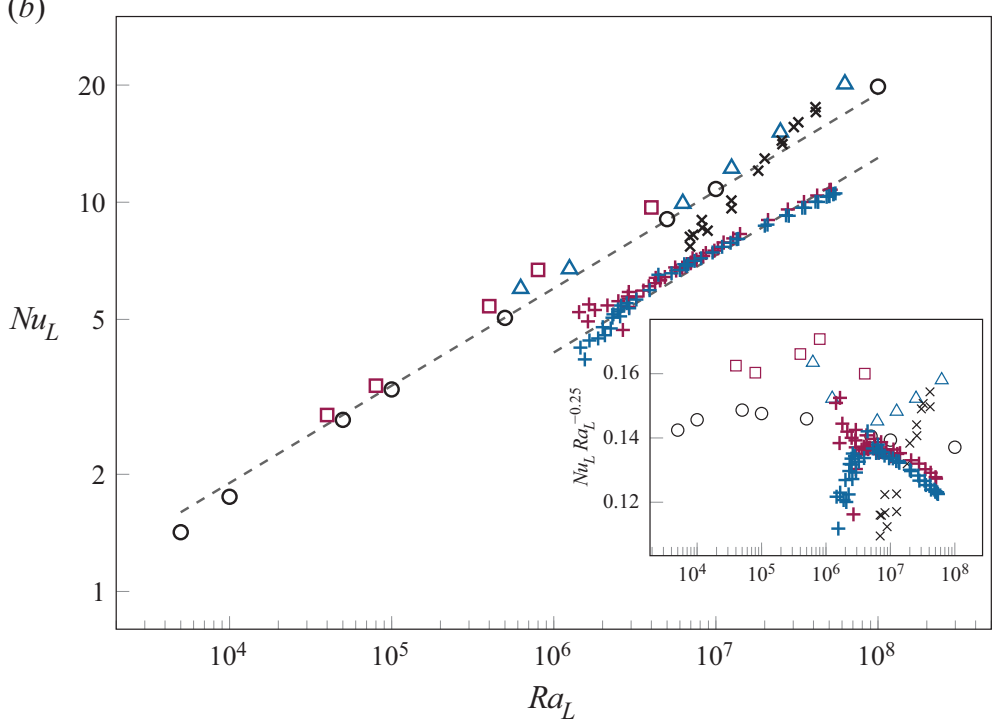

Figure 4. Nusselt number, $N u$, vs Rayleigh number, $R a$, based on length scales: (a) distance of hot and cold plate $H$ and $(b)$ plate length $L$. For $\Gamma=3$ the Nusselt number is measured at the hot plate (red pluses) and at the cold plate (blue pluses). The insets show the compensated Nusselt number based on a $R a^{1 / 4}$-scaling (indicated by dashed lines).

A comparison of the length scales for the Nusselt number is shown in figure 4(a,b) and the data collapse for the length scale $L$, while they do not do so for length scale $H$. In general, one can see this collapse for the Reynolds numbers in figure 5(a,b) and the BL thickness in figure 6. This means, that the relevant length scale in $\mathrm{VC}$ is the plate size, $L$, rather than the plate distance, $H$, for global quantities like $N u$ and $R e$. Further below, will analyse these quantities and figures in detail.

The Nusselt number for the $\Gamma=3$ experiments is only $\approx 70 \%$ of the value observed in the numerical simulations. Interestingly, this appears to be a systematic difference in liquid 
(a)
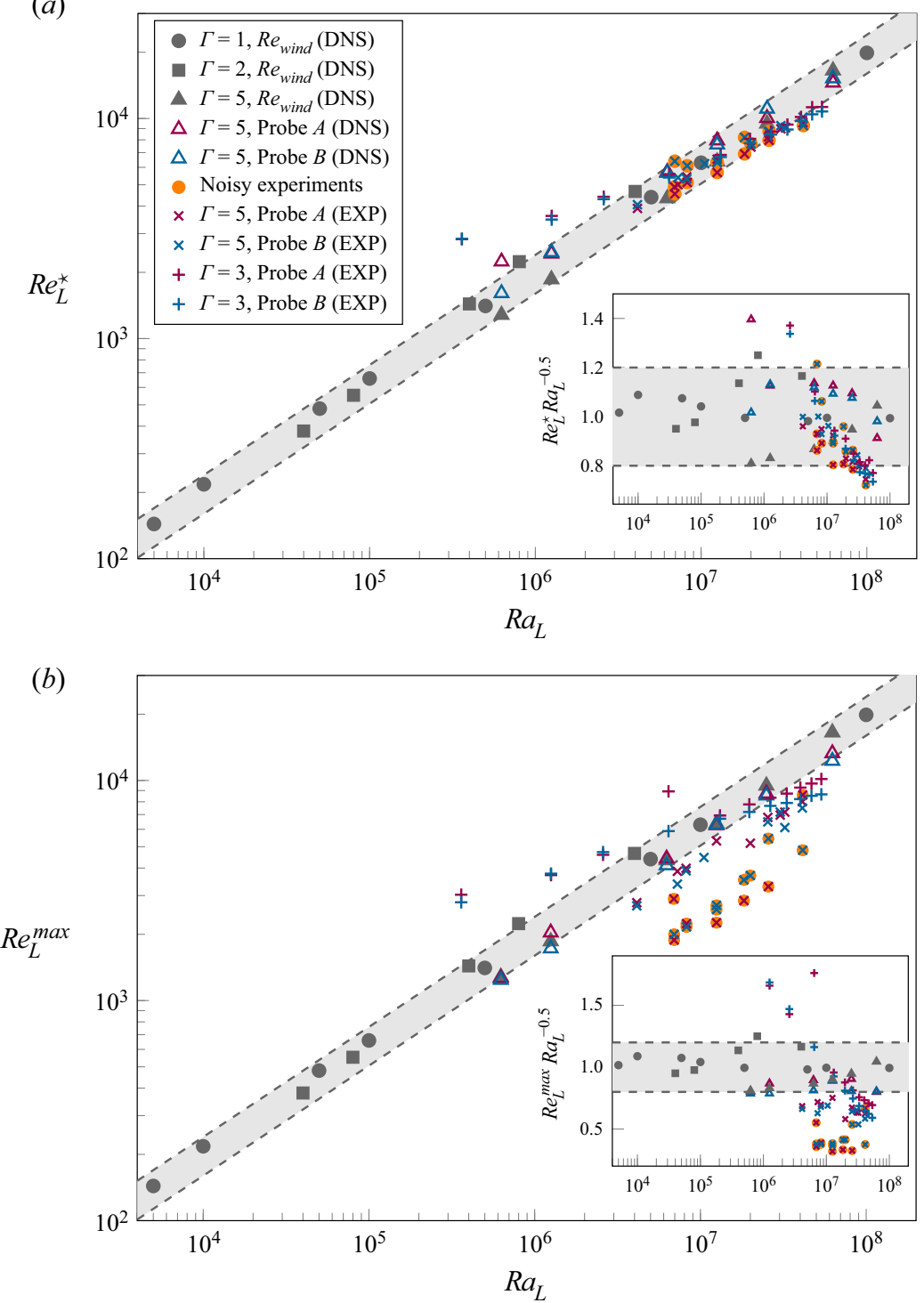

Figure 5. (a) Reynolds numbers based on the characteristic velocity $R e^{\star}$, and (b) Reynolds numbers based on the maximal vertical velocity vs the Rayleigh number. The data of red/blue triangles (DNS) and crosses/pluses (experiments) are obtained by probes at position $A / B$, respectively. The orange background of data points indicates noisy measurements, cf. figure $2(e)$. The dashed lines show the theoretical scaling $R e \sim R a^{1 / 2}$ (Shishkina 2016) and the grey band represents the uncertainty margin of $\pm 20 \%$. The wind-based Reynolds numbers $R e_{\text {wind }}$ from DNS data are shown by grey solid symbols for different aspect ratios $\Gamma=1$ (circle), 2 (square) and 5 (triangle). The insets show compensated plots.

metal simulations and experiments. Zwirner et al. (2020a) compare DNS and experiments of VC with liquid sodium as fluid inside a cylinder $(\Gamma=1)$. There, the absolute measurement of $\mathrm{Nu}$ also shows similar differences. Since the DNS results are in agreement with the literature (Scheel \& Schumacher 2016), we think this systematic deviation is due to some physical effects not considered within the Navier-Stokes equations, and should be investigated in the future. 


\section{Zwirner and others}

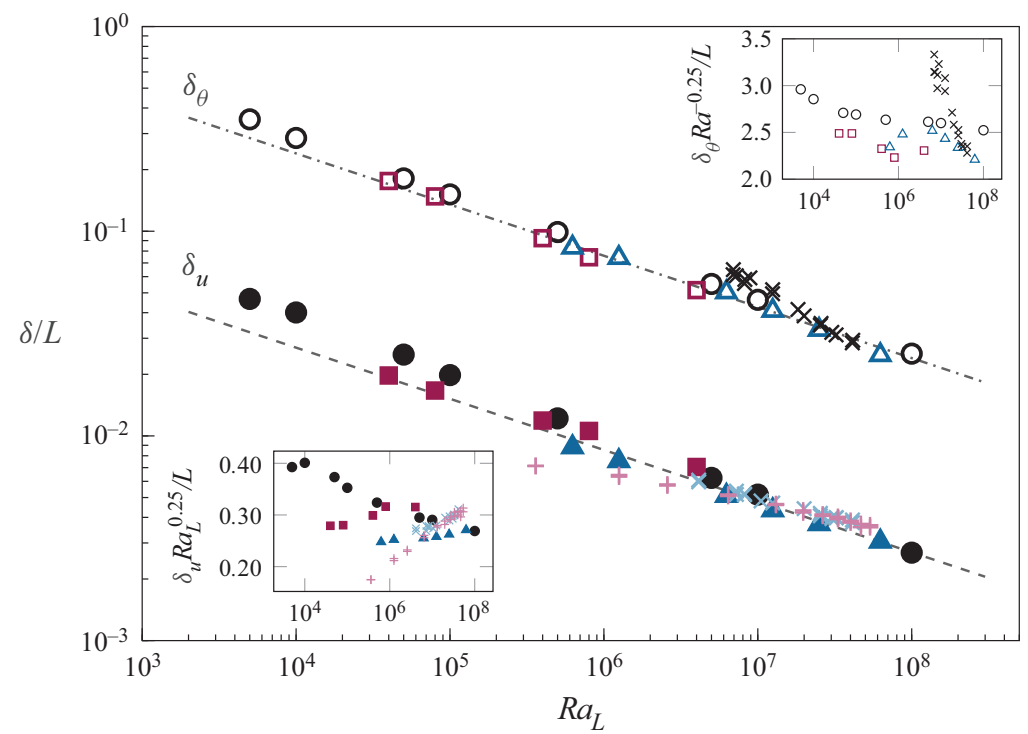

Figure 6. Thermal BL thickness $\delta_{\theta}=H /(2 \mathrm{Nu})$ (open symbols, black crosses) and viscous BL thickness $\delta_{u}$ based on slope criterion (filled symbols, blue crosses, red pluses) vs the Rayleigh number. The dashed and dash-dotted lines show the theoretical scaling law $\delta \sim R a^{-0.25}$ for both BLs. The insets show the compensated data. Experimental data for $\Gamma=3$ (pluses) and $\Gamma=5$ the (crosses). DNS data (circles, squares, triangles) as in figure 4.

As next step, we take a closer look at the local flow organization, which reveals a more complex picture. Here, we focus on the time-averaged heat transport across the central vertical cross-section $\bar{\Omega}_{x}$ (figure $7 a$ ) and the respective profile along the vertical direction (figure $7 b$ ). The profiles are obtained from the same simulations as the instantaneous snapshots in figure 3 . Note that the three red curves correspond to figure $3(d, e, c)$ and have a similar $R a_{L}$. Although, the slopes of the profiles close to the boundary are similar, the behaviour in the bulk is qualitatively different for different aspect ratios. The smaller aspect ratios, $\Gamma=1$ and 2, have a steep rise and a steep fall of the heat transport around its maximum close to the wall, where the heat transport is approximately 5 times larger than the average $N u$, while for $\Gamma=5$, the rise and fall are gentle and the maximum is slightly above the average heat transport. The shape of the heat transport profile for the $\Gamma=5$ case shares some similarities with the profiles at smaller aspect ratios $\Gamma=1$ and 2 for similar $R a_{H}$ (blue curves). Therefore, one may conclude that the local flow organization is strongly influenced by the aspect ratio and the profiles show a rather complex behaviour. This is further supported by the shapes of the time-averaged horizontal profiles of the temperature and vertical velocity component (figure 8).

In figure 9 we show the vertical profiles of the thermal BL thickness at the heated plate, for the same cases discussed in the previous paragraph. Here, one notices that, for the same $R a_{H}$, the profiles are quite different for different aspect ratios (figure $9 a$ ), while, for similar $R a_{L} \approx 5 \times 10^{6}$, the thermal BLs are of similar structure (figure $9 b$ ). At the top $(z / L=0)$ and bottom $(z / L=1)$ one can prominently notice the influence of the adiabatic boundaries. The thermal $\mathrm{BL}$ grows from the bottom to top, first slowly until $z / L \approx 0.7$ and then faster, being influenced by the adiabatic top boundary. 
(a)

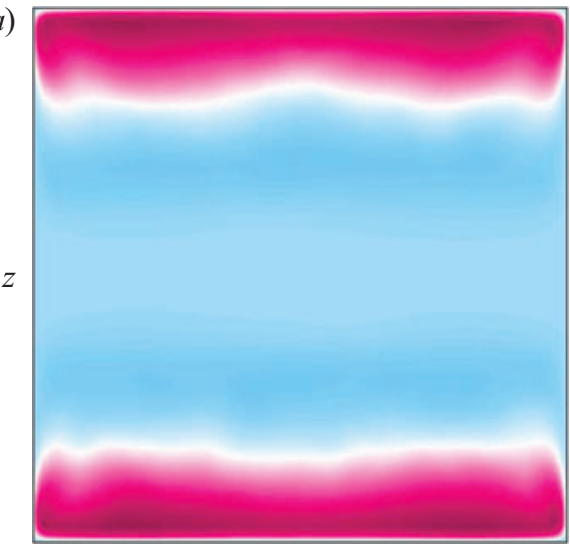

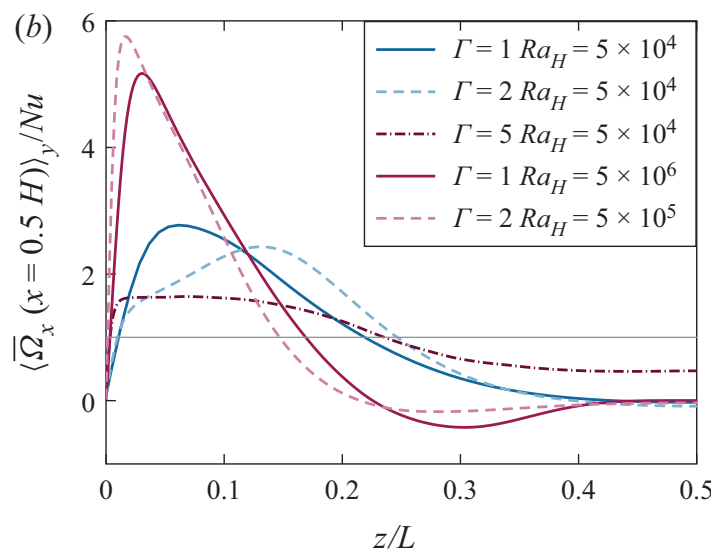

Figure 7. (a) Cross-section of $\bar{\Omega}_{x}(x=0.5 H) / N u$ for $\Gamma=1$ and $R a_{H}=5 \times 10^{6}$ and (b) profiles of the local heat transport $\bar{\Omega}_{x}(x=0.5 H)$ averaged in the $y$-direction as functions of vertical coordinate $z$. For the red lines $R a_{L} \approx 5 \times 10^{6}$ and these data are collected from the same simulations as presented in figure $3(c-e)$. All DNS data.

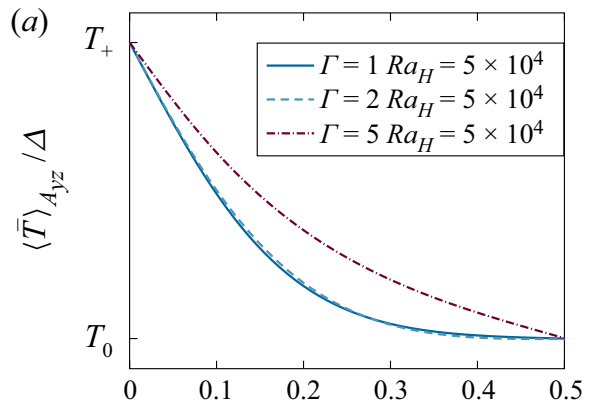

(b)

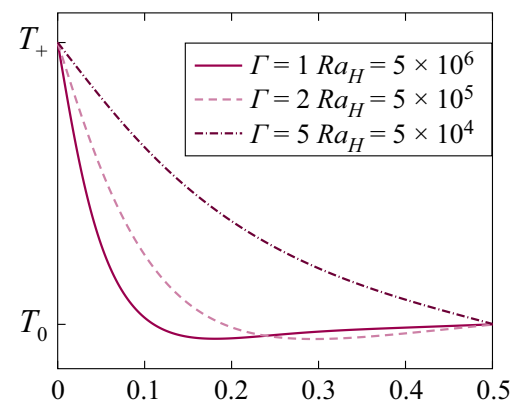

(c)

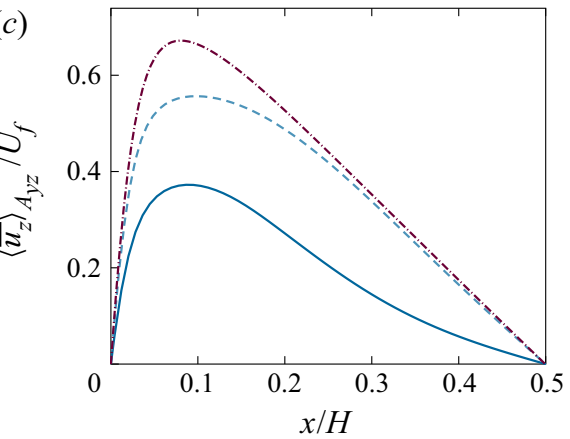

(d)

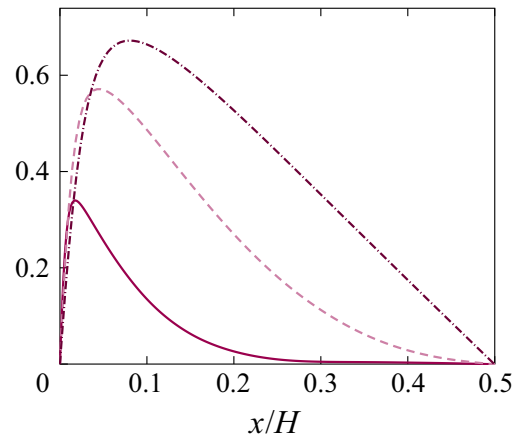

Figure 8. Time-averaged horizontal profiles of $(a, b)$ the temperature and $(c, d)$ the vertical velocity component for $(a, c) R a_{H}=5 \times 10^{4}$ and $(b, d) R a_{L} \approx 5 \times 10^{6}$. All DNS data.

\subsection{The Reynolds number in experiments and DNS}

In $\S 2.4$, we introduced two different methods to extract the Reynolds number and in the following we discuss the robustness of these methods and compare the results from experiments and DNS. We need to take into account that the beam of the 


\section{Zwirner and others}

(a)

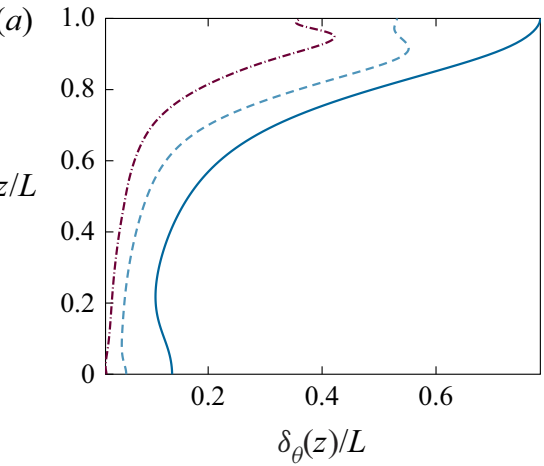

(b)

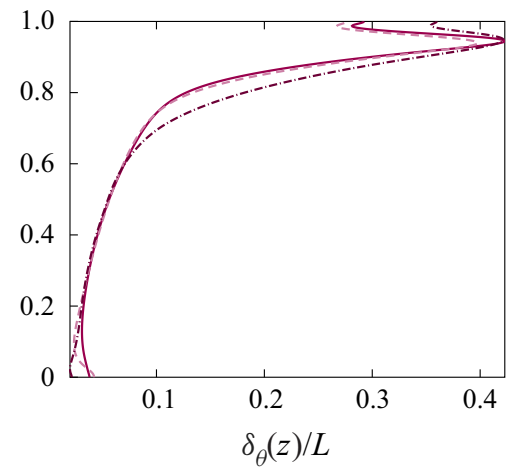

Figure 9. Profiles of the thermal BL $\delta_{\theta} / L=-\left(2 \partial_{x} T\right)^{-1}$ at the hot plate $x=0$ averaged in the $y$-direction as a function of the vertical coordinate $z$. Note that this definition of $\delta_{\theta}$ is analogous to $\delta_{\theta} / L=1 /(2 N u)$, and at the plate heat is transported exclusively by conduction; (a) $\Gamma=1,2,5$ and $R a_{H}=5 \times 10^{4}$ and $(b) \Gamma=1,2,5$ at similar $R a_{L} \approx 5 \times 10^{6}$. The legend is identical to figure $7(b)$. All DNS data.
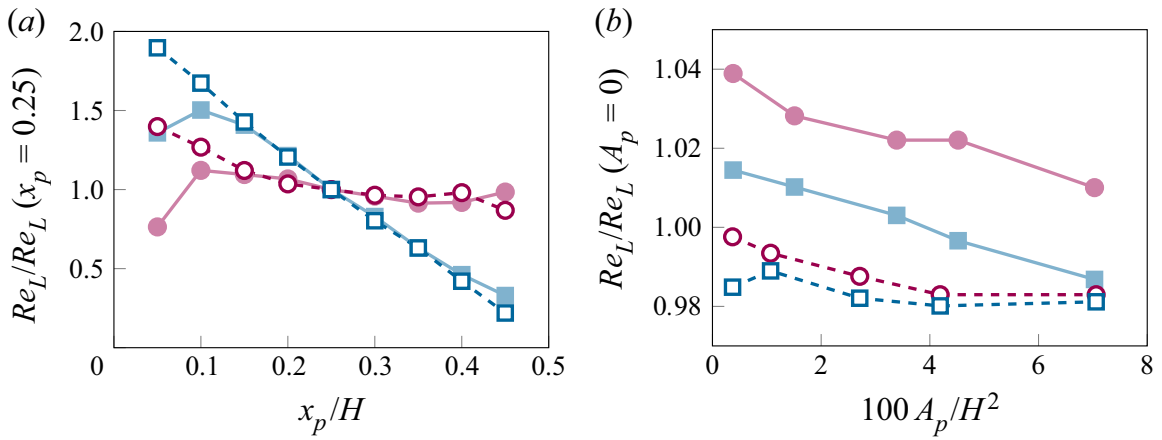

Figure 10. Dependence of the normalized Reynolds numbers $R e_{L}^{\max }$ (squares) and $R e_{L}^{\star}$ (circles) on (a) the horizontal probe position and $(b)$ the beam thickness (represented by the cross-sectional area $A_{p}$ ) for the DNS at $R a_{H}=10^{4}$ (filled symbols), $R a_{H}=5 \times 10^{5}$ (open symbols) and $\Gamma=5$.

Doppler-velocimetry probe has a certain horizontal extent of diameter less than $H / 4$. Analysing the DNS data, we can mimic this horizontal extent by locally averaging over a certain area. Due to the Cartesian geometry used in the simulations, we average over a beam with a square cross-section of area $A_{p}$ and a side length of up to $H / 4$ to account for the finite extent of the Doppler-velocimetry beam.

To verify the robustness of our measurements of the vertical velocity component we separately analyse the influence of two major aspects: (i) the horizontal position of the probe and (ii) the thickness of the ultrasonic beam. The Reynolds number $R e_{L}^{\star}$ depends weakly on the horizontal position $x_{p}$ of the probe, while $R e_{L}^{\max }$ depends strongly on the horizontal position of the probe (figure 10a). By changing the averaging area of the square beam from our numerical probes in the DNS, we find that the extend of the probing beam has a rather small effect of $\approx 2 \%$ on the Reynolds numbers (figure $10 b$ ).

The $R e_{L}^{\star}$ data of regular and noisy experimental data agree very well (figure $5 a$ ), but the $R e_{L}^{m a x}$ data show systematically lower values for noisy experiments (figure $5 b$ ). This demonstrates the second advantage of the proposed method for extracting the Reynolds number, besides the weak dependence on the probe position. Note that this weak dependence on the probe position is quite important, as the positions in the experimental 


\section{Vertical convection of liquid metals}

set-ups for $\Gamma=3$ and 5 differ. The experimental $R e_{L}^{\star}$-data collapse nicely for both aspect ratios (figure $5 a$ ).

From the discussion above, we conclude that $R e_{L}^{\star}$ and thus $U^{\star}$ is an appropriate measure of the characteristic velocity in the system. To finalize this section, we give a physical interpretation of $U^{\star}$. From the definition of $U^{\star}(2.13)$ and figure $2(a, c, e)$ it becomes clear that $U^{\star}$ quantifies the vertical advection component of the velocity fluctuations along the line of measurement, or in other words: it is the advection velocity of the fluctuations.

\subsection{The scaling relations of global $\mathrm{Nu}$ and $\mathrm{Re}$}

In Shishkina (2016) the scaling relations for heat and momentum transport in laminar $\mathrm{VC}$ were derived based on similarity solutions for the BL equations. Furthermore, the validity of this theoretical scaling was shown by DNS data for VC inside a cylindrical domain of aspect ratio one. In figures 4,5 and 6 the theoretical scalings for $N u \sim R a^{1 / 4}$ and $R e \sim R a^{1 / 2}$ are indicated by dashed lines. These results agree well with our DNS and experimental data.

A general remark on the uncertainty of the scaling relations: the Prandtl number in the experiments might be slightly larger than 0.03 and a good estimate is up to $10 \%$, i.e. 0.033 . To ensure that this does not affect our conclusions, we estimate how it would influence the data in the double logarithmic plots. The theoretical scaling from Shishkina (2016) agrees well with our data, hence we use it as the basis for our analysis. The prefactor for the $\mathrm{Nu}$ and $R e$ scalings in our plots thus depends on the Prandtl number, which, for the slightly larger Prandtl number of $\mathrm{Pr}=0.033$ results in a factor of 1.024 and 1.049 , respectively (cf. (1.1)). From this one may conclude that the experimental data of $\mathrm{Nu}$ and $\mathrm{Re}$ might be shifted by $2.4 \%$ and $4.9 \%$ towards higher $\mathrm{Nu}$ and $R e$, respectively, which would provide a even better collapse with the DNS data. Although one should keep in mind that this is by far not the only source of uncertainty in our data, nevertheless this estimation seems to be a reasonable explanation of the systematic deviation between experiments and DNS in the $\mathrm{Nu}$ and $\mathrm{Re}$ data.

One observation from the experiments is that the scaling exponents of $R a$ are slightly larger for the Nusselt number in the case of $\Gamma=5$, but slightly smaller for the Reynolds number compared with the expected scaling exponents from theory and also DNS. The major challenge in the experiments is the measurement of very small temperature differences, especially at small $R a$. Therefore, the determination of $N u$ in this range is highly error prone. From this, we noticed that the uncertainty of Nusselt number is especially large for low Rayleigh numbers and, together with the fact that the experiments cover just one order of magnitude in Rayleigh number, this could lead to a large uncertainty of the scaling exponent.

The deviations of the Reynolds number in the experiments compared with the theoretical scalings one can interpret as follows. With increasing thermal forcing, not only does the amplitude of the velocity increase, but also the flow structure changes in the convection cell. Thus, the location, where the maximum vertical velocity is achieved, moves towards the tempered plates and away from the beam of the UDV measurements. Although the measurement of $R e_{L}^{\star}$ shows only a weak dependence on the probe position (especially for $\Gamma=5$ ), the evaluation of the DNS data for all aspect ratios suggests that the scaling exponent of $R e_{L}^{\star}$ becomes slightly smaller as the velocity is measured further away from the tempered plates. The measuring position of the sensor, however, remains unchanged. It seems to be also plausible that this effect is more strongly manifested for $\Gamma=3$ than for $\Gamma=5$. Unfortunately, in the experiments, we cannot change the sensor position 


\section{Zwirner and others}

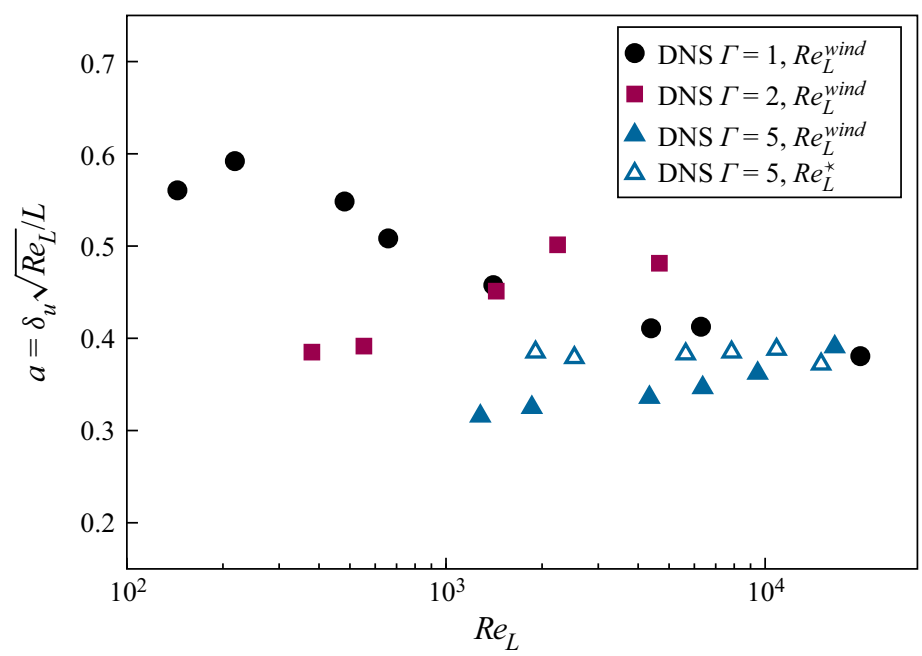

Figure 11. The parameter $a$ from the relation $\delta_{u} / L=a \sqrt{R e}$ (cf. Prandtl 1905) vs the Reynolds number. All data from DNS at different aspect ratios $\Gamma$ for the Reynolds number based on the wind velocity (2.7) and $R e_{L}^{\star}$.

easily, but scenarios are conceivable in which the measurements at a fixed position underestimate the increase in $R e_{L}^{\star}$ with $R a$ and therefore underestimates the scaling exponent.

The thickness of the thermal and viscous BLs can be obtained from $\delta_{\theta} / L=1 /\left(2 N u_{L}\right)$ and $\delta_{u} / L=a / \sqrt{R e_{L}}$ (Prandtl 1905), respectively. In figure 6 we show the respective BLs, and, while the thermal BL thickness is obtained straightforwardly, the viscous BL needs an estimation of the parameter $a$ which is widely accepted in case of RBC to be $\approx 0.482$ for a cylindrical cell of unit aspect ratio (Grossmann \& Lohse 2002), but in general dependent on $\Gamma$. From the DNS data, using $R e_{L}^{\star}$, we can estimate this parameter for $\Gamma=5$ to be $a \approx 0.38$ and then apply this value of $a$ to the experimental data for both aspect ratios $\Gamma=3$ and 5. Thus, we calculate the average ratio of $R e_{L}^{\star-1 / 2}$ and $\delta_{u}$, which is obtained by (2.8). The estimate of $a$ works well for both aspect ratios $\Gamma=3$ and 5 used by the experiments (figure 6). This gives an estimate that the viscous $\mathrm{BL}$ at $R a_{L} \approx 10^{7}$ has a thickness of approximately $1 \mathrm{~mm}$, while the distance between the plates is $H=40 \mathrm{~mm}$ $(\Gamma=5)$.

This procedure can also be applied to the different aspect ratios, and by using $R e_{\text {wind }}$ instead of $R e^{\star}$ as the Reynolds number. A full comparison is shown in figure 11. At first glance these data show no conclusive trend, however, one needs to take into account the above discussed uncertainty margin of $\pm 20 \%$ for the Reynolds numbers. With this in mind, figure 11 is similar to the compensated plots shown as insets of figure $5(a, b)$ and these data show similar scatter.

\section{Conclusions}

We studied VC of a liquid metal with low Prandtl number $(\operatorname{Pr} \approx 0.03)$, more precisely, the eutectic alloy GaInSn, using the complementary results from experimental measurements and DNS. The agreement between DNS and experiments is reasonable and deviations are within the acceptable range. Furthermore, we extensively discussed these deviations. 


\section{Vertical convection of liquid metals}

Our study shows quantitatively, with respect to the global heat and momentum transport, and qualitatively by the instantaneous heat transport superstructures (figure 3), that the plate size $L$, rather than the distance between the plates $H$, is the relevant length scale in VC.

Furthermore, we found that the bulk of the flow contributes only little to the global heat transport. The heat is mainly transported by horizontal layers close to the top/bottom boundary of the convection cell, while the bulk heat transport is far below the mean heat transport or even slightly negative. These heat transporting layers have a thickness in the range of approximately $0.1 L$ to $0.25 L$ each.

Most importantly, we reported a novel method to extract the wind velocity from experimental data, in particular from time-space plots of Doppler-velocimetry measurements, using the two-dimensional autocorrelation defined in (2.12). With the support from the DNS, we show that the Reynolds numbers obtained by our novel method are very similar to the wind-based Reynolds numbers, while they are only weakly dependent on the horizontal position of the probe. Additionally, we showed that even for noisy experimental data our method delivers reasonable Reynolds numbers. Note that this cannot be achieved in direct measurements of the velocity, since they are strongly influenced by the probe position and lead to systematically smaller Reynolds numbers. Further, we report a value of $a \approx 0.38$ to calculate the viscous BL thickness via $\delta_{u}=$ $a L / \sqrt{R e_{L}}$ for VC.

Although the results are obtained only at a single value of $\operatorname{Pr} \approx 0.03$, one would expect similar behaviour for fluids with $\operatorname{Pr} \leq 1$ in general. Altogether, we gained important knowledge on the turbulent wind extraction, in particular in liquid metals. This is important for accurate calculations of the Reynolds number from experimental data and also because the scaling theories (Grossmann \& Lohse 2000; Shishkina 2016) are built upon the notion of wind. One challenge for further theoretical studies is that, in VC, unlike $\mathrm{RBC}$, there is a non-closed term in the exact relation between the global kinetic energy dissipation rate and the vertical convective heat transport ( $\mathrm{Ng}$ et al. 2015; Zwirner \& Shishkina 2018). Also, for the future, a deeper investigation of the local flow organization is necessary, because it, unlike the global quantities, strongly depends on the aspect ratio.

Acknowledgements. The authors acknowledge the Leibniz Supercomputing Centre (LRZ) for providing computing time.

Funding. This work is funded by the Deutsche Forschungsgemeinschaft (DFG) under the grants Sh405/7 (Priority Programme SPP 1881 'Turbulent Superstructures'), VO 2331/1-1 and VO 2331/4-1.

Declaration of interests. The authors report no conflict of interest.

\section{Author ORCIDs.}

(1) Lukas Zwirner https://orcid.org/0000-0002-8805-7292;

(1) Sanjay Singh https://orcid.org/0000-0002-5305-7524;

(1) Sven Eckert https://orcid.org/0000-0003-1639-5417;

(1) Tobias Vogt https://orcid.org/0000-0002-0022-5758;

(1) Olga Shishkina https://orcid.org/0000-0002-6773-6464.

\section{Appendix}

The following tables contain relevant experimental and numerical data. 


\section{Zwirner and others}

DNS resolution

\begin{tabular}{lccccccc}
\hline$L / H$ & $R a_{H}$ & $N_{x}$ & $N_{y}$ & $N_{z}$ & $T_{\text {avg }} / t_{f}$ & $N_{\delta_{u}}$ & $N_{\delta_{\theta}}$ \\
1 & $5 \times 10^{3}$ & 114 & 114 & 114 & 252 & 7 & 41 \\
1 & $1 \times 10^{4}$ & 114 & 114 & 114 & 251 & 6 & 34 \\
1 & $5 \times 10^{4}$ & 114 & 114 & 114 & 252 & 4 & 22 \\
1 & $1 \times 10^{5}$ & 170 & 170 & 170 & 292 & 4 & 27 \\
1 & $5 \times 10^{5}$ & 226 & 226 & 226 & 335 & 4 & 25 \\
1 & $5 \times 10^{6}$ & 338 & 338 & 338 & 161 & 5 & 31 \\
1 & $1 \times 10^{7}$ & 450 & 450 & 450 & 186 & 5 & 35 \\
1 & $1 \times 10^{8}$ & 674 & 674 & 674 & 66 & 7 & 47 \\
2 & $5 \times 10^{3}$ & 114 & 142 & 142 & 326 & 6 & 41 \\
2 & $1 \times 10^{4}$ & 114 & 142 & 142 & 328 & 5 & 35 \\
2 & $5 \times 10^{4}$ & 114 & 170 & 170 & 531 & 5 & 26 \\
2 & $1 \times 10^{5}$ & 170 & 226 & 226 & 332 & 5 & 26 \\
2 & $5 \times 10^{5}$ & 226 & 338 & 338 & 205 & 5 & 26 \\
5 & $5 \times 10^{3}$ & 114 & 226 & 226 & 286 & 6 & 48 \\
5 & $1 \times 10^{4}$ & 114 & 226 & 226 & 286 & 6 & 43 \\
5 & $5 \times 10^{4}$ & 170 & 338 & 338 & 299 & 7 & 49 \\
5 & $1 \times 10^{5}$ & 226 & 450 & 450 & 320 & 6 & 49 \\
5 & $2 \times 10^{5}$ & 226 & 450 & 450 & 162 & 6 & 40 \\
5 & $5 \times 10^{5}$ & 226 & 450 & 450 & 219 & 5 & 30
\end{tabular}

Table 1. Simulation parameters: the aspect ratio $(L / H)$, Rayleigh number $\left(R a_{H}\right)$, grid size $\left(N_{x} \times N_{y} \times N_{z}\right)$, averaging time $\left(T_{a v g}\right)$ and number of points in the viscous $\left(N_{\delta_{u}}\right)$ and thermal BLs $\left(N_{\delta_{\theta}}\right)$. The Prandtl number is fixed to $\operatorname{Pr}=0.03$.

\section{DNS}

\begin{tabular}{lcccccrrr}
\hline$L / H$ & $R a_{H}$ & $R a_{L}$ & $N u_{H}$ & $R e_{L}^{\max }$ & $R e_{L}^{\star}$ & $R e_{\text {wind }}$ & $\delta_{u} / H$ & $\delta_{\theta} / H$ \\
1 & $5 \times 10^{3}$ & $5 \times 10^{3}$ & 1.42 & - & - & 144 & 0.0467 & 0.352 \\
1 & $1 \times 10^{4}$ & $1 \times 10^{4}$ & 1.75 & - & - & 218 & 0.0401 & 0.286 \\
1 & $5 \times 10^{4}$ & $5 \times 10^{4}$ & 2.76 & - & - & 481 & 0.0250 & 0.181 \\
1 & $1 \times 10^{5}$ & $1 \times 10^{5}$ & 3.30 & - & - & 659 & 0.0198 & 0.151 \\
1 & $5 \times 10^{5}$ & $5 \times 10^{5}$ & 5.05 & - & - & 1407 & 0.0122 & 0.099 \\
1 & $5 \times 10^{6}$ & $5 \times 10^{6}$ & 9.05 & - & - & 4391 & 0.0062 & 0.055 \\
1 & $1 \times 10^{7}$ & $1 \times 10^{7}$ & 10.82 & - & - & 6294 & 0.0052 & 0.046 \\
1 & $1 \times 10^{8}$ & $1 \times 10^{8}$ & 19.82 & - & - & 19874 & 0.0027 & 0.025 \\
2 & $5 \times 10^{3}$ & $4 \times 10^{4}$ & 1.42 & - & - & 380 & 0.0395 & 0.352 \\
2 & $1 \times 10^{4}$ & $8 \times 10^{4}$ & 1.69 & - & - & 553 & 0.0333 & 0.296 \\
2 & $5 \times 10^{4}$ & $4 \times 10^{5}$ & 2.70 & - & - & 1437 & 0.0238 & 0.185 \\
2 & $1 \times 10^{5}$ & $8 \times 10^{5}$ & 3.35 & - & - & 2237 & 0.0212 & 0.149 \\
2 & $5 \times 10^{5}$ & $4 \times 10^{6}$ & 4.85 & - & - & 4662 & 0.0141 & 0.103 \\
5 & $5 \times 10^{3}$ & $6.25 \times 10^{5}$ & 1.20 & 1257 & 1908 & 1281 & 0.0441 & 0.417 \\
5 & $1 \times 10^{4}$ & $1.25 \times 10^{6}$ & 1.35 & 1851 & 2525 & 1858 & 0.0377 & 0.371 \\
5 & $5 \times 10^{4}$ & $6.25 \times 10^{6}$ & 1.99 & 4270 & 5638 & 4339 & 0.0255 & 0.252 \\
5 & $1 \times 10^{5}$ & $1.25 \times 10^{7}$ & 2.44 & 6305 & 7849 & 6373 & 0.0217 & 0.205 \\
5 & $2 \times 10^{5}$ & $2.50 \times 10^{7}$ & 3.03 & 8557 & 10858 & 9475 & 0.0186 & 0.165 \\
5 & $5 \times 10^{5}$ & $6.25 \times 10^{7}$ & 4.02 & 12686 & 14974 & 16522 & 0.0152 & 0.124
\end{tabular}

Table 2. Summary of the measured quantities in the DNS for $\operatorname{Pr}=0.03$ : the aspect ratio $(L / H)$, the Rayleigh number $(R a)$ based on $L$ and $H$, the Nusselt number $(N u)$, the various Reynolds numbers ( $R e$ ) defined by (2.11), (2.15) and (2.7) and the viscous and thermal BL thicknesses $\left(\delta_{u}, \delta_{\theta}\right)$. Note that $R e_{L}^{\star}$ and $R e_{L}^{\max }$ are the averages of probes $A$ and $B$. 


\begin{tabular}{lccccc}
\multicolumn{5}{l}{ Experiments } \\
\hline$L / H$ & $R a_{H}$ & $R a_{L}$ & $N u_{H}$ & $R e_{L}^{\max }$ & $R e_{L}^{\star}$ \\
3 & $1.30 \times 10^{4}$ & $3.60 \times 10^{5}$ & - & 2911 & 2836 \\
3 & $4.51 \times 10^{4}$ & $1.25 \times 10^{6}$ & - & 3745 & 3542 \\
3 & $9.29 \times 10^{4}$ & $2.59 \times 10^{6}$ & - & 4661 & 4355 \\
3 & $2.31 \times 10^{5}$ & $6.42 \times 10^{6}$ & - & 7405 & 5488 \\
3 & $4.72 \times 10^{5}$ & $1.31 \times 10^{7}$ & - & 6817 & 6758 \\
3 & $7.09 \times 10^{5}$ & $1.97 \times 10^{7}$ & - & 7489 & 7904 \\
3 & $9.49 \times 10^{5}$ & $2.64 \times 10^{7}$ & - & 8007 & 8590 \\
3 & $1.68 \times 10^{6}$ & $4.68 \times 10^{7}$ & - & 8312 & 9148 \\
3 & $1.19 \times 10^{6}$ & $3.32 \times 10^{7}$ & - & 8755 & 9948 \\
3 & $1.44 \times 10^{6}$ & $4.01 \times 10^{7}$ & - & 9101 & 10850 \\
3 & $1.93 \times 10^{6}$ & $5.36 \times 10^{7}$ & - & 9401 & 11025 \\
5 & $3.30 \times 10^{4}$ & $4.13 \times 10^{6}$ & - & 2740 & 3982 \\
5 & $5.53 \times 10^{4}$ & $6.91 \times 10^{6}$ & 1.63 & 2898 & $4889^{\dagger}$ \\
5 & $5.55 \times 10^{4}$ & $6.94 \times 10^{6}$ & 1.54 & 1924 & $5471^{\dagger}$ \\
5 & $5.80 \times 10^{4}$ & $7.26 \times 10^{6}$ & 1.65 & 3625 & 5197 \\
5 & $6.55 \times 10^{4}$ & $8.19 \times 10^{6}$ & 1.80 & 3939 & 5375 \\
5 & $6.59 \times 10^{4}$ & $8.24 \times 10^{6}$ & 1.72 & 2199 & $5610^{\dagger}$ \\
5 & $7.11 \times 10^{4}$ & $8.89 \times 10^{6}$ & 1.69 & - & - \\
5 & $8.40 \times 10^{4}$ & $1.05 \times 10^{7}$ & - & 4468 & 6236 \\
5 & $9.97 \times 10^{4}$ & $1.25 \times 10^{7}$ & 2.02 & 4011 & $6419^{\dagger}$ \\
5 & $9.99 \times 10^{4}$ & $1.25 \times 10^{7}$ & 1.93 & 2425 & $6015^{\dagger}$ \\
5 & $1.46 \times 10^{5}$ & $1.83 \times 10^{7}$ & 2.41 & 3189 & $7557^{\dagger}$ \\
5 & $1.60 \times 10^{5}$ & $2.00 \times 10^{7}$ & 2.59 & 4443 & $7545^{\dagger}$ \\
5 & $2.04 \times 10^{5}$ & $2.55 \times 10^{7}$ & 2.88 & 6640 & 8468 \\
5 & $2.06 \times 10^{5}$ & $2.57 \times 10^{7}$ & 2.82 & 4370 & $8358^{\dagger}$ \\
5 & $2.42 \times 10^{5}$ & $3.03 \times 10^{7}$ & 3.12 & 7075 & 9129 \\
5 & $2.58 \times 10^{5}$ & $3.23 \times 10^{7}$ & 3.21 & 6647 & 9092 \\
5 & $3.29 \times 10^{5}$ & $4.12 \times 10^{7}$ & 3.51 & 7754 & 9848 \\
5 & $3.31 \times 10^{5}$ & $4.14 \times 10^{7}$ & 3.41 & 6705 & $19784^{\dagger}$
\end{tabular}

Table 3. Summary of the measured quantities in the experiments: the Rayleigh number $(R a)$ based on $L$ and $H$, the Nusselt number $(\mathrm{Nu})$, and the two Reynolds numbers $(R e)$ defined by (2.11) and (2.15). Note that $R e_{L}^{\star}$ and $R e_{L}^{\max }$ are the averages of probes $A$ and $B$. Rows marked by ${ }^{\dagger}$ involve noisy UDV data. The experiments are conducted at $P r \approx 0.03$ and the aspect ratios $L / H=3$ and 5 . 


\section{Zwirner and others}

Experiments $\Gamma=3$

\begin{tabular}{|c|c|c|c|c|c|c|c|}
\hline$R a_{H}$ & $R a_{L}$ & $N u_{H}$ (hot) & $N u_{H}($ cold $)$ & $R a_{H}$ & $R a_{L}$ & $N u_{H}$ (hot) & $N u_{H}($ cold $)$ \\
\hline $5.31 \times 10^{4}$ & $1.43 \times 10^{6}$ & 1.74 & - & $2.36 \times 10^{5}$ & $6.38 \times 10^{6}$ & - & 2.31 \\
\hline $5.41 \times 10^{4}$ & $1.46 \times 10^{6}$ & - & 1.41 & $2.41 \times 10^{5}$ & $6.51 \times 10^{6}$ & 2.28 & - \\
\hline $5.76 \times 10^{4}$ & $1.55 \times 10^{6}$ & - & 1.32 & $2.44 \times 10^{5}$ & $6.60 \times 10^{6}$ & - & 2.29 \\
\hline $6.02 \times 10^{4}$ & $1.62 \times 10^{6}$ & 1.65 & - & $2.50 \times 10^{5}$ & $6.75 \times 10^{6}$ & - & 2.31 \\
\hline $6.11 \times 10^{4}$ & $1.65 \times 10^{6}$ & 1.82 & - & $2.58 \times 10^{5}$ & $6.98 \times 10^{6}$ & 2.32 & - \\
\hline $6.15 \times 10^{4}$ & $1.66 \times 10^{6}$ & - & 1.47 & $2.60 \times 10^{5}$ & $7.02 \times 10^{6}$ & 2.33 & - \\
\hline $6.65 \times 10^{4}$ & $1.79 \times 10^{6}$ & 1.76 & - & $2.66 \times 10^{5}$ & $7.18 \times 10^{6}$ & 2.39 & - \\
\hline $6.96 \times 10^{4}$ & $1.88 \times 10^{6}$ & - & 1.49 & $2.74 \times 10^{5}$ & $7.39 \times 10^{6}$ & - & 2.36 \\
\hline $7.43 \times 10^{4}$ & $2.01 \times 10^{6}$ & - & 1.59 & $2.81 \times 10^{5}$ & $7.58 \times 10^{6}$ & 2.37 & - \\
\hline $7.66 \times 10^{4}$ & $2.07 \times 10^{6}$ & - & 1.52 & $3.00 \times 10^{5}$ & $8.11 \times 10^{6}$ & - & 2.39 \\
\hline $7.94 \times 10^{4}$ & $2.14 \times 10^{6}$ & 1.81 & - & $3.09 \times 10^{5}$ & $8.35 \times 10^{6}$ & 2.43 & - \\
\hline $8.32 \times 10^{4}$ & $2.25 \times 10^{6}$ & - & 1.58 & $3.21 \times 10^{5}$ & $8.67 \times 10^{6}$ & 2.47 & - \\
\hline $8.52 \times 10^{4}$ & $2.30 \times 10^{6}$ & - & 1.68 & $3.53 \times 10^{5}$ & $9.54 \times 10^{6}$ & - & 2.48 \\
\hline $8.63 \times 10^{4}$ & $2.33 \times 10^{6}$ & - & 1.72 & $3.62 \times 10^{5}$ & $9.79 \times 10^{6}$ & 2.50 & - \\
\hline $9.22 \times 10^{4}$ & $2.49 \times 10^{6}$ & - & 1.77 & $3.82 \times 10^{5}$ & $1.03 \times 10^{7}$ & 2.54 & - \\
\hline $9.26 \times 10^{4}$ & $2.50 \times 10^{6}$ & - & 1.75 & $4.05 \times 10^{5}$ & $1.09 \times 10^{7}$ & - & 2.56 \\
\hline $9.27 \times 10^{4}$ & $2.50 \times 10^{6}$ & 1.86 & - & $4.13 \times 10^{5}$ & $1.12 \times 10^{7}$ & 2.63 & - \\
\hline $9.51 \times 10^{4}$ & $2.57 \times 10^{6}$ & - & 1.70 & $4.56 \times 10^{5}$ & $1.23 \times 10^{7}$ & - & 2.62 \\
\hline $9.64 \times 10^{4}$ & $2.60 \times 10^{6}$ & - & 1.81 & $4.74 \times 10^{5}$ & $1.28 \times 10^{7}$ & 2.69 & - \\
\hline $9.88 \times 10^{4}$ & $2.67 \times 10^{6}$ & 1.57 & - & $4.98 \times 10^{5}$ & $1.34 \times 10^{7}$ & - & 2.68 \\
\hline $1.04 \times 10^{5}$ & $2.82 \times 10^{6}$ & - & 1.83 & $5.09 \times 10^{5}$ & $1.37 \times 10^{7}$ & - & 2.68 \\
\hline $1.06 \times 10^{5}$ & $2.86 \times 10^{6}$ & 1.91 & - & $5.22 \times 10^{5}$ & $1.41 \times 10^{7}$ & 2.76 & - \\
\hline $1.07 \times 10^{5}$ & $2.89 \times 10^{6}$ & 1.88 & - & $7.42 \times 10^{5}$ & $2.00 \times 10^{7}$ & - & 2.90 \\
\hline $1.07 \times 10^{5}$ & $2.90 \times 10^{6}$ & 1.96 & - & $7.71 \times 10^{5}$ & $2.08 \times 10^{7}$ & - & 2.92 \\
\hline $1.08 \times 10^{5}$ & $2.91 \times 10^{6}$ & 1.79 & - & $7.77 \times 10^{5}$ & $2.10 \times 10^{7}$ & 3.00 & - \\
\hline $1.08 \times 10^{5}$ & $2.92 \times 10^{6}$ & - & 1.78 & $1.00 \times 10^{6}$ & $2.71 \times 10^{7}$ & - & 3.08 \\
\hline $1.19 \times 10^{5}$ & $3.23 \times 10^{6}$ & 1.91 & - & $1.03 \times 10^{6}$ & $2.78 \times 10^{7}$ & 3.19 & - \\
\hline $1.20 \times 10^{5}$ & $3.23 \times 10^{6}$ & - & 1.87 & $1.04 \times 10^{6}$ & $2.82 \times 10^{7}$ & - & 3.08 \\
\hline $1.32 \times 10^{5}$ & $3.57 \times 10^{6}$ & 1.98 & - & $1.26 \times 10^{6}$ & $3.41 \times 10^{7}$ & - & 3.23 \\
\hline $1.43 \times 10^{5}$ & $3.86 \times 10^{6}$ & 2.03 & - & $1.29 \times 10^{6}$ & $3.50 \times 10^{7}$ & 3.34 & - \\
\hline $1.45 \times 10^{5}$ & $3.90 \times 10^{6}$ & - & 1.98 & $1.32 \times 10^{6}$ & $3.55 \times 10^{7}$ & - & 3.23 \\
\hline $1.56 \times 10^{5}$ & $4.22 \times 10^{6}$ & 2.06 & - & $1.52 \times 10^{6}$ & $4.12 \times 10^{7}$ & - & 3.34 \\
\hline $1.58 \times 10^{5}$ & $4.25 \times 10^{6}$ & 2.13 & - & $1.56 \times 10^{6}$ & $4.21 \times 10^{7}$ & 3.47 & - \\
\hline $1.64 \times 10^{5}$ & $4.42 \times 10^{6}$ & - & 2.17 & $1.58 \times 10^{6}$ & $4.27 \times 10^{7}$ & - & 3.34 \\
\hline $1.68 \times 10^{5}$ & $4.53 \times 10^{6}$ & 2.10 & - & $1.79 \times 10^{6}$ & $4.82 \times 10^{7}$ & - & 3.44 \\
\hline $1.68 \times 10^{5}$ & $4.53 \times 10^{6}$ & 2.11 & - & $1.85 \times 10^{6}$ & $5.00 \times 10^{7}$ & - & 3.46 \\
\hline $1.79 \times 10^{5}$ & $4.85 \times 10^{6}$ & 2.14 & - & $1.85 \times 10^{6}$ & $5.01 \times 10^{7}$ & - & 3.46 \\
\hline $1.97 \times 10^{5}$ & $5.33 \times 10^{6}$ & - & 2.19 & $1.86 \times 10^{6}$ & $5.02 \times 10^{7}$ & 3.59 & - \\
\hline $2.08 \times 10^{5}$ & $5.61 \times 10^{6}$ & 2.27 & - & $1.89 \times 10^{6}$ & $5.12 \times 10^{7}$ & 3.59 & - \\
\hline $2.11 \times 10^{5}$ & $5.69 \times 10^{6}$ & 2.23 & - & $1.97 \times 10^{6}$ & $5.31 \times 10^{7}$ & - & 3.49 \\
\hline $2.22 \times 10^{5}$ & $6.00 \times 10^{6}$ & - & 2.23 & $1.97 \times 10^{6}$ & $5.32 \times 10^{7}$ & - & 3.50 \\
\hline $2.30 \times 10^{5}$ & $6.20 \times 10^{6}$ & 2.27 & - & $2.02 \times 10^{6}$ & $5.47 \times 10^{7}$ & - & 3.51 \\
\hline
\end{tabular}

Table 4. Experiments with $\Gamma=3$ : the Rayleigh number $(R a)$ based on $L$ and $H$, the Nusselt number $(N u)$ based on $H$ measured at the hot or cold plate. 


\section{Vertical convection of liquid metals}

\section{REFERENCES}

Ahlers, G., Brown, E. \& Nikolaenko, A. 2006 The search for slow transients, and the effect of imperfect vertical alignment, in turbulent Rayleigh-Bénard convection. J. Fluid Mech. 557, 347-367.

Ahlers, G., Grossmann, S. \& Lohse, D. 2009 Heat transfer and large scale dynamics in turbulent Rayleigh-Bénard convection. Rev. Mod. Phys. 81, 503-537.

Aubert, J., Brito, D., NATAF, H.-C., CARDin, P. \& MAsson, J.-P. 2001 A systematic experimental study of rapidly rotating spherical convection in water and liquid gallium. Phys. Earth Planet. Inter. 128 (1-4), $51-74$.

Aurnou, J.M., Bertin, V., Grannan, A.M., Horn, S. \& Vogt, T. 2018 Rotating thermal convection in liquid gallium: multi-modal flow, absent steady columns. J. Fluid Mech. 846, 846-876.

BATCHELOR, G.K. 1954 Heat transfer by free convection across a closed cavity between vertical boundaries at different temperatures. Q. Appl. Maths 12, 209-233.

BEJAN, A. 1980 A synthesis of analytical results for natural convection heat transfer across rectangular enclosures. Intl J. Heat Mass Transfer 23 (5), 723-726.

BEJAN, A. 1985 Mass and heat transfer by natural convection in a vertical cavity. Intl J. Heat Fluid Flow 6 (3), $149-159$.

BeJAn, A. 2013 Convection Heat Transfer, 4th edn. John Wiley \& Sons.

Belmonte, A., Tilgner, A. \& Libchaber, A. 1995 Turbulence and internal waves in side-heated convection. Phys. Rev. E 51, 5681-5687.

Bodenschatz, E., Pesch, W. \& Ahlers, G. 2000 Recent developments in Rayleigh-Bénard convection. Annu. Rev. Fluid Mech. 32, 709-778.

Braunsfurth, M.G., Skeldon, A.C., Juel, A., Mullin, T. \& Riley, D.S. 1997 Free convection in liquid gallium. J. Fluid Mech. 342, 295-314.

Brito, D., NATAF, H.-C., CARdin, P., Aubert, J. \& MAsson, J.-P. 2001 Ultrasonic Doppler velocimetry in liquid gallium. Exp. Fluids 31 (6), 653-663.

Chen, Y.-M. \& Pearlstein, A.J. 1989 Stability of free-convection flows of variable-viscosity fluids in vertical and inclined slots. J. Fluid Mech. 198, 513-541.

Chillà, F., Rastello, M., Chaumat, S. \& Castaing, B. 2004 Long relaxation times and tilt sensitivity in Rayleigh-Bénard turbulence. Eur. Phys. J. B 40 (2), 223-227.

Chillà, F. \& Schumacher, J. 2012 New perspectives in turbulent Rayleigh-Bénard convection. Eur. Phys. $J$. E 35, 58.

Churchill, S.W. \& ChU, H.H.S. 1975 Correlating equations for laminar and turbulent free convection from a vertical plate. Intl J. Heat Mass Transfer 18, 1323-1329.

DANiEls, K.E., WienER, R.J. \& BODENSCHATZ, E. 2003 Localized transverse bursts in inclined layer convection. Phys. Rev. Lett. 91, 114501.

ECKert, S. \& Gerbeth, G. 2002 Velocity measurements in liquid sodium by means of ultrasound Doppler velocimetry. Exp. Fluids 32 (5), 542-546.

Fujit, T., TAkeuchi, M., FujiI, M., Suzaki, K. \& Uehar, H. 1970 Experiments on natural-convection heat transfer from the outer surface of a vertical cylinder to liquids. Intl J. Heat Mass Transfer 13, 753-770.

GILL, A.E. 1966 The boundary-layer regime for convection in a rectangular cavity. J. Fluid Mech. 26, 515-536.

Gillet, N., BRito, D., JAUlt, D. \& NATAF, H.C. 2007 Experimental and numerical studies of convection in a rapidly rotating spherical shell. J. Fluid Mech. 580, 83-121.

Graebel, W.P. 1981 The influence of Prandtl number on free convection in a rectangular cavity. Intl J. Heat Mass Transfer 24, 125-131.

Grossmann, S. \& LohSE, D. 2000 Scaling in thermal convection: a unifying theory. J. Fluid Mech. 407, $27-56$.

Grossmann, S. \& Lohse, D. 2002 Prandtl and Rayleigh number dependence of the Reynolds number in turbulent thermal convection. Phys. Rev. E 66, 016305.

Kelley, D.H. \& WeIER, T. 2018 Fluid mechanics of liquid metal batteries. Appl. Mech. Rev. 70 (2), 020801.

King, E.M. \& AURnOU, J.M 2013 Turbulent convection in liquid metal with and without rotation. Proc. Natl Acad. Sci. USA 110, 6688-6693.

Kis, P. \& HeRwig, H. 2012 The near wall physics and wall functions for turbulent natural convection. Intl J. Heat Mass Transfer 55, 2625-2635.

Kooij, G.L., Botchev, M.A., Frederix, E.M.A., Geurts, B.J., Horn, S., Lohse, D., van der Poel, E.P., Shishrina, O., Stevens, R.J.A.M. \& Verzicco, R. 2018 Comparison of computational codes for direct numerical simulations of turbulent Rayleigh-Bénard convection. Comput. Fluids 166, 1-8.

Koster, J.N., Seidel, T. \& Derebail, R. 1997 A radioscopic technique to study convective fluid dynamics in opaque liquid metals. J. Fluid Mech. 343, 29-41. 


\section{Zwirner and others}

Nataf, H.-C., Alboussiere, T., Brito, D., Cardin, P., Gagnière, N., Jault, D. \& Schmitt, D. 2008 Rapidly rotating spherical Couette flow in a dipolar magnetic field: an experimental study of the mean axisymmetric flow. Phys. Earth Planet. Inter. 170 (1-2), 60-72.

NG, C.S., Ooi, A., Lohse, D. \& Chung, D. 2015 Vertical natural convection: application of the unifying theory of thermal convection. J. Fluid Mech. 764, 349-361.

Ostrach, S. 1972 Natural convection in enclosures. Adv. Heat Transfer 8, 161-227.

Pallares, J., Vernet, A., Ferre, J.A. \& Grau, F.X. 2010 Turbulent large-scale structures in natural convection vertical channel flow. Intl J. Heat Mass Transfer 53 (19), 4168-4175.

PAOLUCCI, S. 1990 Direct numerical simulation of two-dimensional turbulent natural convection in an enclosed cavity. J. Fluid Mech. 215, 229-262.

PAOluCCI, S. 1994 The differentially heated cavity. Sadhana 19 (5), 619-647.

Plevachuk, Y., Sklyarchuk, V., Eckert, S., Gerbeth, G. \& Novakovic, R. 2014 Thermophysical properties of the liquid Ga-In-Sn eutectic alloy. J. Chem. Engng Data 59 (3), 757-763.

PRANDTL, L. 1905 Über Flüssigkeitsbewegung bei sehr kleiner Reibung. In Verhandlungen des III. Int. Math. Kongr., Heidelberg, 1904, pp. 484-491. Teubner.

Saville, D.A. \& Churchill, S.W. 1969 Free Convection at low Prandtl numbers in laminar boundary layers. Ind. Engng Chem. Fund. 8 (2), 329-332.

ScheEl, J.D. \& SCHUMACHER, J. 2016 Global and local statistics in turbulent convection at low Prandtl numbers. J. Fluid Mech. 802, 147-173.

Schumacher, J., Bandaru, V., Pandey, A. \& Scheel, J.D. 2016 Transitional boundary layers in low-Prandtl-number convection. Phys. Rev. Fluids 1, 084402.

ShishkinA, O. 2016 Momentum and heat transport scalings in laminar vertical convection. Phys. Rev. E 93, 051102.

ShishrinA, O. 2021 Rayleigh-Bénard convection: the container shape matters. Phys. Rev. Fluids 6, 090502.

ShishkinA, O. \& HoRn, S. 2016 Thermal convection in inclined cylindrical containers. J. Fluid Mech. 790, R3.

SUN, C., XI, H.-D. \& XIA, K.-Q. 2005 Azimuthal symmetry, flow dynamics, and heat transport in turbulent thermal convection in a cylinder with an aspect ratio of 0.5. Phys. Rev. Lett. 95, 074502.

TAKEDA, Y. 2012 Ultrasonic Doppler Velocity Profiler for Fluid Flow. Springer.

TSUJI, T. \& NAGANO, Y. 1988 Characteristics of a turbulent natural convection boundary layer along a vertical flat plate. Intl J. Heat Mass Transfer 31 (8), 1723-1734.

Versteegh, T.A.M. \& Nieuwstadt, F.T.M. 1999 A direct numerical simulation of natural convection between two infinite vertical differentially heated walls scaling laws and wall functions. Intl J. Heat Mass Transfer 42, 3673-3693.

Vogt, T., Horn, S. \& Aurnou, J.M. $2021 a$ Oscillatory thermal-inertial flows in liquid metal rotating convection. J. Fluid Mech. 911, A5.

Vogt, T., Horn, S., Grannan, A.M. \& Aurnou, J.M. $2018 a$ Jump rope vortex in liquid metal convection. Proc. Natl Acad. Sci. USA 115, 12674-12679.

Vogt, T., Ishimi, W., Yanagisawa, T., Tasaka, Y., Sakuraba, A. \& Eckert, S. $2018 b$ Transition between quasi-two-dimensional and three-dimensional Rayleigh-Bénard convection in a horizontal magnetic field. Phys. Rev. Fluids 3, 013503.

Vogt, T., RÄBIGER, D. \& ECKERT, S. 2014 Inertial wave dynamics in a rotating liquid metal. J. Fluid Mech. 753, 472-498.

Vogt, T., YAng, J.-C., Schindler, F. \& ECKert, S. $2021 b$ Free-fall velocities and heat transport enhancement in liquid metal magneto-convection. J. Fluid Mech. 915, A68.

Wang, Q., LiU, H.-R., Verzicco, R., Shishinina, O. \& Lohse, D. 2021 Regime transitions in thermally driven high-Rayleigh number vertical convection. J. Fluid Mech. 917, A6.

WEI, T. 2020 Scaling of Reynolds stresses in a differentially heated vertical channel. Phys. Rev. Fluids 4, 051501.

WeISS, S. \& AHLERS, G. 2013 Effect of tilting on turbulent convection: cylindrical samples with aspect ratio $\gamma=0.50$. J. Fluid Mech. 715, 314-334.

YAng, J.C., VogT, T. \& ECKERT, S. 2021 Transition from steady to oscillating convection rolls in Rayleigh-Bénard convection under the influence of a horizontal magnetic field. Phys. Rev. Fluids 6 (2), 023502.

ZHOU, Q. \& XIA, K.-Q. 2010 Measured instantaneous viscous boundary layer in turbulent Rayleigh-Bénard convection. Phys. Rev. Lett. 104, 104301.

Zürner, T., Schindler, F., Vogt, T., Eckert, S. \& Schumacher, J. 2019 Combined measurement of velocity and temperature in liquid metal convection. J. Fluid Mech. 876, 1108-1128. 


\section{Vertical convection of liquid metals}

Zürner, T., Schindler, F., Vogt, T., Eckert, S. \& Schumacher, J. 2020 Flow regimes of Rayleigh-Bénard convection in a vertical magnetic field. J. Fluid Mech. 894, A21.

Zwirner, L., Khalilov, R., Kolesnichenko, I., Mamykin, A., Mandrykin, S., Pavlinov, A., Shestakov, A., Teimurazov, A., Frick, P. \& Shishrina, O. $2020 a$ The influence of the cell inclination on the heat transport and large-scale circulation in liquid metal convection. J. Fluid Mech. 884, A18.

Zwirner, L. \& Shishinina, O. 2018 Confined inclined thermal convection in low-Prandtl-number fluids. J. Fluid Mech. 850, 984-1008.

Zwirner, L., Tilgner, A. \& Shishrina, O. $2020 \mathrm{~b}$ Elliptical instability and multiple-roll flow modes of the large-scale circulation in confined turbulent Rayleigh-Bénard convection. Phys. Rev. Lett. 125, 054502. 